\title{
UNIFORMIZATION OF ANALYTIC SPACES
}

\author{
EDWARD BIERSTONE AND PIERRE D. MILMAN
}

\section{Contents}

1. Introduction

2. Analytic spaces and blowings-up

3. The hypersurface case

4. The diagram of initial exponents

5. The Hilbert-Samuel function

6. Essential variables of a homogeneous ideal

7. The effect of blowing up

8. Embedded uniformization theorems Bibliography

\section{INTRODUCTION}

In this article, we give an explicit elementary proof of a local version of resolution of singularities in characteristic zero. "Local" means that the centres of blowing up are chosen locally, so that a finite number of finite sequences of local blowings-up may be required to cover a neighbourhood of a given point. (Hence we use the term "uniformization" rather than "local desingularization," although uniformization in our sense is considerably stronger than the original idea of Zariski [16].) In the last two decades, several mathematicians (notably Abhyankar [1], Hironaka and Spivakovsky [12], and Youssin [14, 15]) have proposed simpler or more explicit versions of the inductive procedure in $\mathrm{Hi}$ ronaka's proof of his great theorem [8]. These approaches would seem to lead to some form of uniformization, though full details of none of them have yet appeared. The idea of seeking an explicit local procedure to determine the centres of blowing up is that a sufficiently good local choice should globalize automatically. (Our method, for example, gives global resolution of singularities of surfaces in any codimension.)

Throughout this paper, $\mathbf{K}$ denotes either the field of real numbers $\mathbf{R}$ or the field of complex numbers $\mathbf{C}$. We will work in the category of analytic spaces over $\mathbf{K}$, although our techniques apply as well to algebraic spaces over a field

Received by the editors April 11, 1989.

1980 Mathematics Subject Classification (1985 Revision). Primary 14E15, 32C45; Secondary 14B05, 32B30.

Research of the first author was partially supported by NSERC operating grant A9070.

Research of the second author was partially supported by NSERC operating grant A8849. 
of characteristic zero. Let $M$ be a smooth analytic space. A family of analytic mappings $\left\{\pi_{j}: M_{j} \rightarrow M\right\}$ will be said to form a locally finite covering of $M$ if:

(1) The images $\pi_{j}\left(M_{j}\right)$ are subordinate to a locally finite covering of $M$ by open subsets.

(2) If $K$ is a compact subset of $M$, then there are compact subsets $K_{j}$ of $M_{j}$ such that $K=\bigcup \pi_{j}\left(K_{j}\right)$. (The union is finite, by (1).)

Theorem 1.1 (Embedded uniformization). Let $X$ be a closed analytic subspace of $M$. Suppose that $X$ is reduced. Then there is a locally finite covering $\left\{\pi_{j}: M_{j} \rightarrow M\right\}$ of $M$ such that:

(1) Each $\pi_{j}$ is a composite of finitely many local blowing-ups with smooth centres in Hilbert-Samuel strata of the successive strict transforms of $X$.

(2) For each $j$, let $X_{j}$ denote the (final) strict transform of $X$ by $\pi_{j}$. Then:

(i) $X_{j}$ is a smooth analytic subspace of $M_{j}$;

(ii) $\pi_{j}^{-1}(X)=X_{j} \cup \pi_{j}^{-1}(\operatorname{Sing} X)$, as sets;

(iii) $X_{j}$ and $\pi_{j}^{-1}(\operatorname{Sing} X)$ simultaneously have only normal crossings.

The terms used in Theorem 1.1 (which are standard in works on resolution of singularities) will be defined in the course of the paper. The singular subspace Sing $X$ of $X$ denotes the smallest analytic subspace outside of which $X$ is smooth. Conditions (1) and (2)(ii) of the theorem imply that each $\pi_{j}^{-1}(\operatorname{Sing} X)$ (as a set) is a hypersurface given by the union of the inverse images of the centres of all the local blowing-ups which make up $\pi_{j}$. In particular, $\pi_{j}$ restricts to an open embedding

$$
X_{j}-\pi_{j}^{-1}(\operatorname{Sing} X) \rightarrow X-\operatorname{Sing} X \text {. }
$$

Condition (2)(iii) means that, locally, each $M_{j}$ admits a coordinate system $\left(x_{1}, \ldots, x_{n}\right)$ in which $\pi_{j}^{-1}(\operatorname{Sing} X)$ is given by a monomial equation $x_{1}^{\alpha_{1}} \cdots x_{n}^{\alpha_{n}}$ $=0$, and $X_{j}$ is defined by the equations $x_{i}=0$, for certain $i$.

We will, in fact, prove a result stronger than Theorem 1.1 which applies to analytic spaces that are not necessarily reduced and specifies more precisely the centres of blowing up (Theorem 8.6). Local finiteness of the covering $\left\{\pi_{j}\right\}$ is a direct consequence of our explicit local description of the centre.

The Hilbert-Samuel function was first used by Bennet [4] and Hironaka [9] as a local measure of singularity. Let $\mathscr{O}=\mathscr{O}_{M}$ denote the sheaf of germs of analytic functions on $M$, and let $\mathscr{I}_{X}$ denote the coherent sheaf of ideals in $\mathscr{O}$ which determines $X$ (cf. $\S 2$ ). The Hilbert-Samuel function $H_{X, x}$ associated to $X$ at the point $x$ is defined as

$$
H_{X, x}(k)=\operatorname{dim}_{\mathbf{K}} \frac{\mathscr{O}_{x}}{\mathscr{I}_{X, x}+\mathfrak{m}_{x}^{k+1}}, \quad k \in \mathbf{N},
$$

where $\mathfrak{m}_{x}$ denotes the maximal ideal of $\mathscr{O}_{x} . \quad(\mathbf{N}$ always denotes the nonnegative integers.) As a function of $x, H_{X, x}$ is upper semicontinuous in the 
analytic Zariski topology (Corollary 5.1.3). The Hilbert-Samuel stratum of $X$ containing a point $x_{0}$ is

$$
S=\left\{x \in X: H_{X, x}=H_{X, x_{0}}\right\} .
$$

The strategy is to cover a neighbourhood of $x_{0}$ by finitely many finite sequences of local blowings-up as in Theorem 1.1(1), with respect to which the HilbertSamuel functions of the strict transforms of $X$ at $x_{0}$ necessarily decrease $(\S \S 7$ and 8 ).

Our theorem will be proved first in the case that $X$ is a hypersurface, in $\S 3$. We formulate a new resolution problem for principal divisors and obtain the hypersurface case (Theorem 3.17) as a consequence of a solution of this general problem (Theorem 3.2). When $X$ is a hypersurface, $\mathscr{I}_{X}$ is principal. In this case, the Hilbert-Samuel stratum $S$ coincides with the equimultiple locus of $X$ through $x_{0},\left\{x \in X: \mu\left(\mathscr{I}_{X, x}\right)=\mu\left(\mathscr{I}_{X, x_{0}}\right)\right\}$, where $\mu\left(\mathscr{I}_{X, x}\right)$ denotes the order (or multiplicity) of $\mathscr{I}_{X, x}$.

$\S \S 4-8$ (which can be read independently of $\S 3$ ) are devoted to proving our main theorem by a reduction to the hypersurface case. Hironaka's proof of resolution of singularities [8] does not simplify in the case of a hypersurface because the inductive procedure involves passing to higher codimension. In [11], Hironaka introduced a method to reduce the general problem to the hypersurface case by describing $X$, in a neighbourhood of a given point, as the intersection of certain special hypersurfaces whose strict transforms can be traced until the Hilbert-Samuel function of $X$ decreases. We realize this scheme in a different way. Our approach involves three important technical ideas:

(1) The "diagram of initial exponents" associated to an ideal in a ring of formal power series $(\S 4)$; in particular, the relationship between differential properties of the ideal and combinatorial properties of the diagram. What we call the "standard basis" of an ideal (Corollary 4.2.1), which is obtained using the diagram and Hironaka's division algorithm (Theorem 4.1.1), is a stronger notion than "standard basis" in the sense used by Hironaka and other authors, and obeys a particularly simple law of transformation by blowing up with suitable centre when the Hilbert-Samuel function does not decrease (Theorem 7.3).

(2) Variation of the diagram in analytically parametrized families of ideals (first studied in [5]). Upper semicontinuity of the diagram with respect to the analytic Zariski topology (Theorem 4.3.2) is elementary, and immediately implies semicontinuity of the Hilbert-Samuel function ( $\$ 5.1)$. Our techniques provide a direct identification of the Hilbert-Samuel stratum and the equimultiple locus of the standard basis $(\S 5.3)$.

(3) Our elementary notion of "essential variables" of a homogeneous ideal (§6). The essential variables of the initial ideal of $\mathscr{I}_{X, x_{0}}$ and their relationship with the Hilbert-Samuel stratum of $X$ containing $x_{0}(\S 7)$ in some sense replaces Hironaka's theory of "maximal contact."

This article is self-contained apart from our use of the division algorithm and of semicontinuity of the diagram of initial exponents. Nevertheless, the reader might find that our earlier work on transforming an analytic function to normal crossings by blowings-up $[7, \S 4]$ helps to motivate the proof of the hypersurface 
case. We express our gratitude to the mathematicians (particularly S. S. Abhyankar, H. Hironaka, M. Spivakovsky, B. Youssin) with whom we have had conversations which helped to clarify our ideas on resolution of singularities.

\section{ANALYTIC SPACES AND BLOWINGS-UP}

Throughout this section, $M$ denotes a smooth analytic space (over $\mathbf{K}$ ), i.e., an analytic manifold (over $\mathbf{K}$ ) regarded as a ringed space $\left(|M|, \mathscr{O}_{M}\right)$, where $|M|$ is the underlying point set of $M$ and $\mathscr{O}=\mathscr{O}_{M}$ denotes the sheaf of germs of analytic functions on $M$.

2.1. Analytic spaces and normal crossings. An open (analytic) subspace $U$ of $M$ is a ringed subspace $\left(|U|, \mathscr{O}_{U}\right)$, where $|U|$ is an open subset of $|M|$ and $\mathscr{O}_{U}$ denotes the restriction of $\mathscr{O}$ to $|U|$. A closed analytic subspace $X$ of $M$ is a ringed subspace $\left(|X|, \mathscr{O}_{X}\right)$ determined by a coherent sheaf of ideals $\mathscr{I}$ in $\mathscr{O}$; i.e., $|X|$ is the support of $\mathscr{O} / \mathscr{I}$ and $\mathscr{O}_{X}$ is the restriction to $|X|$ of $\mathscr{O} / \mathscr{I}$. We write $\mathscr{I}_{X}$ for $\mathscr{I}$. We will usually not distinguish in notation between $X$ and its underlying point set $|X|$. Let $x_{0} \in X$. We say that $X$ is smooth at $x_{0}$ if $x_{0}$ admits a neighbourhood in $M$ with coordinates $\left(x_{1}, \ldots, x_{n}\right)$ such that $\mathscr{I}_{X, x_{0}}$ is generated by some of the $x_{i}$. Thus $X$ is smooth if and only if it is smooth at every point. The singular subspace $\operatorname{Sing} X$ of $X$ denotes the smallest closed analytic subspace $Z$ of $X$ such that $X$ is smooth at every point of $X-Z$.

A closed analytic subspace $X$ of $M$ is said to be reduced if, for all $x \in X$, $\mathscr{O}_{X, x}$ contains no nilpotents. Let $X$ be a closed analytic subspace of $M$. Let $X_{\text {red }}$ denote the smallest closed analytic subspace $Y$ of $X$ such that $|Y|=|X|$. Clearly, $X_{\text {red }}$ is reduced. If $\mathbf{K}=\mathbf{C}$, then $X_{\text {red }}$ is the unique reduced analytic subspace $Y$ of $X$ such that $|Y|=|X|$. If $\mathbf{K}=\mathbf{R}$, there is not, in general, a unique reduced analytic subspace $Y$ such that $|Y|=|X|$.

Let $\mathscr{I}$ be a coherent sheaf of ideals in $\mathscr{O}$, and let $X$ be the closed analytic subspace of $M$ determined by $\mathscr{I}$. We say that $\mathscr{I}$ is a principal divisor (or that $X$ is a hypersurface) if $\mathscr{I}_{x}$ is principal for all $x \in M$. A hyperplane means a smooth hypersurface. Suppose that $\mathscr{F}$ and $\mathscr{J}$ are principal divisors. We say that $\mathscr{I}$ is divisible by $\mathscr{J}$ if there is a principal divisor $\mathscr{H}$ such that $\mathscr{I}=\mathscr{H} \cdot \mathscr{J}$. We write $\mathscr{H}=\mathscr{I} \cdot \mathscr{J}^{-1}$. (Whenever we write this expression, it is implicit that $\mathscr{J}$ is divisible by $\mathscr{J}$.)

Let $\mathscr{I} \subset \mathscr{O}$ be a principal divisor, and let $X$ be the hypersurface determined by $\mathscr{I}$. Let $x_{0} \in M$. We say that $\mathscr{I}$ or $X$ has only normal crossings at $x_{0}$ if $M$ admits a local coordinate system $\left(x_{1}, \ldots, x_{n}\right)$ centered at $x_{0}$ in which $\mathscr{I}_{x_{0}}$ is generated by a monomial $x_{1}^{\alpha_{1}} \cdots x_{n}^{\alpha_{n}}$. Let $Z$ be a closed subspace of $M$. We say that $Z$ and $X$ (or $Z$ and $\mathscr{I}$ ) simultaneously have only normal crossings at $x_{0}$ if $M$ admits a local coordinate system centered at $x_{0}$ in which $\mathscr{F}_{x_{0}}$ is generated by a monomial and $\mathscr{I}_{Z, x_{0}}$ is generated by a family of monomials (therefore, by some of the $x_{i}$ in the case that $Z$ is smooth at $x_{0}$ ). When the conditions above hold at every point of $M$, we say more briefly, "has only normal crossings" or "simultaneously have only normal crossings." 
Let $N$ be a smooth analytic space and let $\phi: N \rightarrow M$ be an analytic mapping (morphism of analytic spaces). Let $\mathscr{I} \subset \mathscr{O}_{M}$ be a coherent sheaf of ideals, and let $X$ denote the corresponding analytic subspace of $M$. Then $\phi^{-1}(\mathscr{I})$ denotes the coherent sheaf of ideals in $\mathscr{O}_{N}$ generated by $\mathscr{I}$ via $\phi$ (i.e., generated by the pull-back $\left.\phi^{*}(\mathscr{I})\right)$ and $\phi^{-1}(X)$ denotes the corresponding analytic subspace of $N$; i.e., $\left|\phi^{-1}(X)\right|=\phi^{-1}(|X|)$ and $\mathscr{O}_{\phi^{-1}(X)}$ is the restriction to $\left|\phi^{-1}(X)\right|$ of $\mathscr{O}_{N} / \phi^{-1}(\mathscr{F})$. If $y \in N$, then $\mathscr{O}_{\phi^{-1}(X), y}=\mathscr{O}_{X, \phi(y)} \otimes_{\mathscr{O}_{M, \phi(y)}} \mathscr{O}_{N, y}$.

2.2. Zariski semicontinuity. Let $X$ be an analytic subset of $M$ and let $\Sigma$ be a partially ordered set. A function $\tau: X \rightarrow \Sigma$ is upper semicontinuous in the analytic Zariski topology of $X$ (Zariski semicontinuous, for short) if:

(1) $\tau(x)$ takes only finitely many values, for $x$ in a compact subset of $X$.

(2) For each $x_{0} \in X,\left\{x \in X: \tau(x) \geq \tau\left(x_{0}\right)\right\}$ is a closed analytic subset of $X$.

2.3. The order of an ideal or of a function. Let $\mathscr{I}$ be a coherent sheaf of ideals in $\mathscr{O}$. Let $x \in M$ and $\mathfrak{m}_{x}$ denote the maximal ideal of $\mathscr{O}_{x}$. The order $\mu\left(\mathscr{F}_{x}\right)$ of $\mathscr{I}$ at $x$ is the largest integer $\mu$ such that $\mathscr{I}_{x} \subset \mathfrak{m}_{x}^{\mu}$.

Remark 2.3.1. (As a function $M \rightarrow \mathbf{N}) \mu\left(\mathcal{F}_{x}\right)$ is Zariski semicontinuous.

Suppose that $U$ is an open subspace of $M$ and that $f \in \mathscr{O}(U)$. Let $x \in U$. The $\operatorname{order}$ (or multiplicity) $\mu_{x}(f)$ of $f$ at $x$ likewise means the largest $\mu$ such that the germ of $f$ at $x$ belongs to $\mathfrak{m}_{x}^{\mu}$. (We put $\mu_{x}(f)=\infty$ if the germ of $f$ at $x$ is 0 .) Suppose that $C$ is a smooth closed analytic subspace of $U$ and that $x \in C$. The order $\mu_{C, x}(f)$ of $f$ along $C$ at $x$ is the largest $\mu$ such that the germ of $f$ at $x$ belongs to $\mathscr{I}_{C, x}^{\mu}$.

Remark 2.3.2. Let $x_{0} \in C$. Clearly, $\mu_{C, x_{0}}(f)$ is the generic value of $\mu_{x}(f)$ for $x$ in a small neighbourhood of $x_{0}$ in $C$. Since $\mu_{x}(f)$ is upper semicontinuous, $\mu_{C, x_{0}}(f)=\mu_{x_{0}}(f)$ if and only if $\mu_{x}(f)$ is constant on $C$ near $x_{0}$.

2.4. Blowing up and local blowing up. For each positive integer $m$, let $P^{m-1}(\mathbf{K})$ denote the $((m-1)$-dimensional $)$ projective space of lines through the origin in $\mathbf{K}^{m}$.

Let $V$ be an open neighbourhood of 0 in $\mathbf{K}^{m}$. Put

$$
V^{\prime}=\left\{(x, l) \in V \times P^{m-1}(\mathbf{K}): x \in l\right\},
$$

and let $\pi: V^{\prime} \rightarrow V$ denote the mapping $\pi(x, l)=x$. Then $\pi$ is proper, $\pi$ restricts to a homeomorphism over $V-\{0\}$, and $\pi^{-1}(0)=P^{m-1}(\mathbf{K})$. The mapping $\pi: V^{\prime} \rightarrow V$ is called the blowing-up of $V$ with centre $\{0\}$.

In a natural way, $V^{\prime}$ is a smooth (algebraic) subspace of $V \times P^{m-1}(\mathbf{K})$ : Let $\left(x_{1}, \ldots, x_{m}\right)$ denote the affine coordinates of $\mathbf{K}^{m}$, and let $\xi=\left[\xi_{1}, \ldots, \xi_{m}\right]$ denote the homogeneous coordinates of $P^{m-1}(\mathbf{K})$. Then

$$
V^{\prime}=\left\{(x, \xi) \in V \times P^{m-1}(\mathbf{K}): x_{i} \xi_{j}=x_{j} \xi_{i}, i, j=1, \ldots, m\right\} .
$$


We can cover $V^{\prime}$ by coordinate charts

$$
V_{i}^{\prime}=\left\{(x, \xi) \in V^{\prime}: \xi_{i} \neq 0\right\}, \quad i=1, \ldots, m,
$$

with coordinates $\left(x_{i 1}, \ldots, x_{i m}\right)$, for each $i$, where

$$
x_{i i}=x_{i}, \quad x_{i j}=\xi_{j} / \xi_{i}, \quad j \neq i .
$$

With respect to these local coordinates, $\pi$ is given by

$$
x_{i}=x_{i i}, \quad x_{j}=x_{i i} x_{i j}, \quad j \neq i .
$$

Suppose that $n>m$ and that $W$ is an open subspace of $\mathbf{K}^{n-m}$. Then the mapping $\pi \times$ id : $V^{\prime} \times W \rightarrow V \times W$ is called the blowing-up of $V \times W$ with centre $\{0\} \times W$.

In the same way, if $C$ is a smooth closed analytic subspace of $M$, we define the blowing-up $\pi: M^{\prime} \rightarrow M$ with centre $C: M^{\prime}$ is a smooth analytic space and $\pi$ is a proper analytic mapping such that:

(1) $\pi$ restricts to an isomorphism $M^{\prime}-\pi^{-1}(C) \rightarrow M-C$.

(2) Let $U \subset M$ be a chart with coordinates given by an analytic isomorphism $\phi: U \rightarrow V \times W$, where $V, W$ are open neighbourhoods of the origins in $\mathbf{K}^{m}, \mathbf{K}^{n-m}$ (respectively), and $\phi(C \cap U)=\{0\} \times W$. Let $\pi_{0}: V^{\prime} \rightarrow V$ be the blowing-up of $V$ with centre $\{0\}$. Then there is an isomorphism $\phi^{\prime}: \pi^{-1}(U) \rightarrow V^{\prime} \times W$ such that the following diagram commutes:

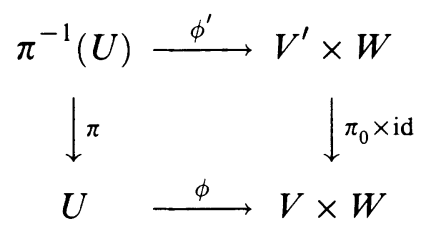

Conditions (1) and (2) above define $\pi: M^{\prime} \rightarrow M$ uniquely, up to an isomorphism of $M^{\prime}$ commuting with $\pi$.

Suppose that $U$ is an open subspace of $M$ and that $C$ is a smooth closed analytic subspace of $U$. Let $\pi: U^{\prime} \rightarrow M$ denote the composition of the blowing-up $U^{\prime} \rightarrow U$ with centre $C$, and the inclusion $U \hookrightarrow M$. We call $\pi$ a local blowing-up of $M$ (over $U$, with smooth centre $C$ ).

We will consider mappings $\pi: N \rightarrow M$ obtained as the composition of a finite sequence of local blowings-up; i.e., $\pi=\pi_{1} \circ \pi_{2} \circ \cdots \circ \pi_{k}$, where, for each $i=1, \ldots, k, \pi_{i}: U_{i+1} \rightarrow U_{i}$ is a local blowing-up of $U_{i}$, and $U_{1}=M$, $U_{k+1}=N$.

2.5. The strict transform. Let $\pi: M^{\prime} \rightarrow M$ be a local blowing-up over an open subspace $U$ of $M$ with smooth centre $C \subset U$. Let $X$ be a closed analytic subspace of $M$.

First suppose that $X$ is a hypersurface. Let $x_{0} \in U$. There is a neighbourhood $V$ of $x_{0}$ in $U$ in which $\mathscr{J}_{X}$ is generated by an analytic function $f(x)$. Suppose that $x_{0} \in C$. Let $\mu=\mu_{C, x_{0}}(f)$. Then we can assume that $V$ has coordinates $x=(w, z), w=\left(w_{1}, \ldots, w_{n-r}\right), z=\left(z_{1}, \ldots, z_{r}\right)$, in which $C$ is given by $z=0$, and that $\mu_{C, x}(f)=\mu$ for all $x \in C \cap V$. Over $V, \pi$ restricts 
to the blowing-up $\pi: V^{\prime} \rightarrow V$ of $V$ with centre $C \cap V$. Then $V^{\prime}=\bigcup_{i=1}^{r} V_{i}^{\prime}$, where, for each $i, V_{i}^{\prime}$ has a coordinate system $\left(w^{\prime}, z^{\prime}\right), w^{\prime}=\left(w_{1}^{\prime}, \ldots, w_{n-r}^{\prime}\right)$, $z^{\prime}=\left(z_{1}^{\prime}, \ldots, z_{r}^{\prime}\right)$, in which $\pi$ is given by $w=w^{\prime}, z_{i}=z_{i}^{\prime}$, and $z_{j}=z_{i}^{\prime} z_{j}^{\prime}$ if $j \neq i$. The strict transform $X^{\prime}$ of $X$ by $\pi$ is defined in $V^{\prime}$ as $X^{\prime}=\bigcup_{i=1}^{r} X_{i}^{\prime}$, where $X_{i}^{\prime}$ is the closed analytic subspace of $V_{i}^{\prime}$ such that $\mathscr{I}_{X_{i}^{\prime}}$ is generated in $V_{i}^{\prime}$ by

$$
f^{\prime}\left(w^{\prime}, z^{\prime}\right)=\frac{1}{\left(z_{i}^{\prime}\right)^{\mu}} f\left(w^{\prime}, z_{i}^{\prime} z_{1}^{\prime}, \ldots, z_{i}^{\prime}, \ldots, z_{i}^{\prime} z_{r}^{\prime}\right) .
$$

(If $x_{0} \notin C$, then we can assume $C \cap V=\varnothing$. Then $\pi$ restricts to an isomorphism $\pi: V^{\prime} \rightarrow V$ over $V$, and $\mathscr{I}_{X^{\prime}}$ is generated in $V^{\prime}$ by $f^{\prime}=f \circ \pi$.)

We also call (the germ at $x_{0}^{\prime} \in \pi^{-1}\left(x_{0}\right)$ of) $f^{\prime}$ the strict transform of (the germ at $x_{0}$ of) $f$; of course, $f^{\prime} \in \mathscr{O}_{M^{\prime}, x_{0}^{\prime}}$ is well-defined only up to multiplication by an invertible factor.

In general, let $x_{0} \in U$ and let $x_{0}^{\prime} \in \pi^{-1}\left(x_{0}\right)$. Then the strict transform $X^{\prime}$ of $X$ by $\pi$ is defined near $x_{0}^{\prime}$ as the intersection of the strict transforms of all the hypersurfaces containing $X$ near $x_{0}$; i.e., $\mathcal{I}_{X^{\prime}}, x_{0}^{\prime}$ is the ideal generated by the strict transforms $f^{\prime}$ of all $f \in \mathscr{I}_{X, x_{0}}$. We also call $\mathscr{I}_{X^{\prime}}$ the strict transform of the ideal sheaf $\mathscr{I}_{X}$.

The strict transform $X^{\prime}$ of $X$ by $\pi$ is the smallest closed analytic subspace of $\pi^{-1}(X)$ such that $\pi$ induces an isomorphism $X^{\prime}-\pi^{-1}(C) \rightarrow X-C$ over $U$. (We will not use this fact.)

We also define the strict transform of $X$ by a finite sequence of local blowingsup with smooth centres, by iterating the definition above.

\section{THE HYPERSURFACE CASE}

Let $M$ be a smooth analytic space (over $\mathbf{K}$ ). We consider data of the following form:

$$
\left(\left(\mathscr{I}_{p}\right)_{p=1, \ldots, s} ; \mathscr{J} ; \mathscr{H}\right)
$$

where $\mathscr{J}_{1}, \ldots, \mathscr{I}_{s}, \mathscr{J}$, and $\mathscr{H}$ are principal divisors on $M$, and $\mathscr{H}$ has only normal crossings. Let $\mu$ be a positive integer. Let $U$ be an open subspace of $M$, and let $C$ be a smooth closed subspace of $U$.

Definition 3.1. We say that $C$ is $\mu$-admissible (as a centre of local blowing-up over $U$ ) if:

(1) $C$ and $\mathscr{H}$ simultaneously have only normal crossings.

(2) $|C| \subset\left\{x \in U: \mu\left(\mathscr{I}_{p, x}\right) \geq \mu, p=1, \ldots, s\right\}$.

Let $\pi: U^{\prime} \rightarrow M$ denote the local blowing-up of $M$ over $U$ with centre $C$. If $C$ is $\mu$-admissible, then we can define a transformation

$$
\left(\left(\mathscr{I}_{p}^{\prime}\right)_{p=1, \ldots, s} ; \mathscr{J}^{\prime} ; \mathscr{H}^{\prime}\right)
$$


of the above data by $\pi$ as follows: Let $\mathscr{K}$ denote the ideal sheaf of $\pi^{-1}(C)$ in $\mathscr{O}_{U^{\prime}}$. Clearly, each $\pi^{-1}\left(\mathscr{I}_{p}\right)$ is divisible by $\mathscr{K}^{\mu}$. Put

$$
\begin{aligned}
\mathscr{J}_{p}^{\prime} & =\pi^{-1}\left(\mathscr{J}_{p}\right) \cdot \mathscr{K}^{-\mu}, \quad p=1, \ldots, s, \\
\mathscr{J}^{\prime} & =\pi^{-1}(\mathscr{J}), \\
\mathscr{H}^{\prime} & =\pi^{-1}(\mathscr{H}) \cdot \mathscr{K}^{\mu} .
\end{aligned}
$$

By Definition 3.1(1), $\mathscr{H}^{\prime}$ has only normal crossings. Therefore, we can likewise define a transformation of the given data by a sequence of local blowings-up whose centres are $\mu$-admissible with respect to the successive transforms of the given data.

Theorem 3.2. Let $\left(\left(\mathscr{J}_{p}\right)_{p=1, \ldots, s} ; \mathscr{J} ; \mathscr{H}\right)$ be as above. Let $\mu$ be a positive integer. Then there is a locally finite covering $\left\{\pi_{j}: M_{j} \rightarrow M\right\}$ of $M$ such that:

(1) Each $\pi_{j}$ is a composite of finitely many local blowings-up with smooth centres which are $\mu$-admissible with respect to the successive transforms of $\left(\left(\mathscr{I}_{p}\right) ; \mathscr{J} ; \mathscr{H}\right)$.

(2) For each $j$, let $\left(\left(\mathscr{I}_{j p}\right) ; \mathscr{J}_{j} ; \mathscr{H}_{j}\right)$ denote the (final) transform of $\left(\left(\mathscr{I}_{p}\right)\right.$; $\mathscr{J} ; \mathscr{H})$ by $\pi_{j}$. Then $\mathscr{J}_{j}$ and $\mathscr{H}_{j}$ simultaneously have only normal crossings at every point $x^{\prime}$ where $\mu\left(\mathscr{F}_{j p, x^{\prime}}\right) \geq \mu, p=1, \ldots, s$.

Definition 3.3. Let $x \in M$. Let $\nu\left(\mathscr{J}_{x}\right)$ denote the order of the ideal in $\mathscr{O}_{x}=$ $\mathscr{O}_{M, x}$ obtained by dividing $\mathscr{J}_{x}$ by all factors in common with $\mathscr{H}_{x}$. Precisely: Let $f$ and $D$ be generators of $\mathscr{J}_{x}$ and $\mathscr{H}_{x}$, respectively. Put $\nu\left(\mathscr{J}_{x}\right)=$ $\min \left\{\mu_{x}(g): f=\prod_{i=1}^{r} l_{i} \cdot g\right.$, where $g \in \mathscr{O}_{x}$ and, for each $i=1, \ldots, r, l_{i} \in \mathrm{m}_{x}$ and $l_{i}$ divides $\left.D\right\}$. (Take $\nu\left(\mathscr{J}_{x}\right)=0$ if $\mathscr{J}_{x}=\mathscr{O}_{x}$.)

Remark 3.4. $\nu\left(\mathscr{J}_{x}\right)$ is invariant under local analytic coordinate changes. As a function of $x \in M, \nu\left(\mathscr{J}_{x}\right)$ is Zariski semicontinuous (cf. §2.2).

Remark 3.5. Locally, all the notions involved in Theorem 3.2 can be translated into the language of analytic functions. Theorem 3.2 is local in nature. We will use the following notation in the proof. Let $f_{p}(x), p=1, \ldots, s, f(x)$, and $D(x)$ denote analytic functions which generate $\mathscr{I}_{p}, p=1, \ldots, s, \mathscr{J}$, and $\mathscr{H}$, respectively, at every point of some open subspace $U$ of $M$. Suppose that $U$ has a coordinate system $x=\left(x_{1}, \ldots, x_{n}\right)$. Let $I \subset\{1, \ldots, n\}$, and let $C$ be the smooth subspace of $U$ determined by the ideal sheaf in $\mathscr{O}_{U}$ generated by the $x_{k}, k \in I$. Let $\pi: U^{\prime} \rightarrow M$ denote the local blowing-up over $U$ with centre $C$. Then $U^{\prime}$ is covered by coordinate charts $U_{k}^{\prime}, k \in I$, such that, for each $k \in I, \pi_{k}=\pi \mid U_{k}^{\prime}$ is given, in coordinates $y=\left(y_{1}, \ldots, y_{n}\right)$ of $U_{k}^{\prime}$, by

$$
\begin{aligned}
& x_{l}=y_{l}, \quad l \notin I, \\
& x_{k}=y_{k}, \\
& x_{l}=y_{k} y_{l}, \quad l \in I-\{k\} .
\end{aligned}
$$


If $C$ is $\mu$-admissible, then $\mathscr{F}_{p}^{\prime}, p=1 \ldots, s, \mathscr{J}^{\prime}$, and $\mathscr{H}^{\prime}$ are generated in $U_{k}^{\prime}$ by the following transforms of $f_{p}, p=1, \ldots, s, f$, and $D$ (respectively):

$$
\begin{aligned}
f_{p}^{\prime}(y) & =f_{p}\left(\pi_{k}(y)\right) \cdot y_{k}^{-\mu}, \quad p=1, \ldots, s, \\
f^{\prime}(y) & =f\left(\pi_{k}(y)\right), \\
D^{\prime}(y) & =D\left(\pi_{k}(y)\right) \cdot y_{k}^{\mu} .
\end{aligned}
$$

If $F, G \in \mathscr{O}(U)$ (or $\mathscr{O}_{x}$ ), we will say that $F$ is equivalent to $G$ (and write $F \sim G)$ if $F$ equals $G$ times a factor which is invertible in $\mathscr{O}(U)$ (or $\mathscr{O}_{x}$ ).

Proof of Theorem 3.2. It suffices to work locally with the functions introduced in Remark 3.5. Let $n=\operatorname{dim} M$. We can assume that $f$ is divisible by $f_{1}, \ldots, f_{s}$ and $D$. Let $x_{0} \in U$. Suppose that $f\left(x_{0}\right)=0$ and that $\mu_{x_{0}}\left(f_{p}\right) \geq \mu, p=$ $1, \ldots, s$. Put $d\left(x_{0}\right)=\nu_{x_{0}}(f)$. Using the Weierstrass preparation theorem, we can assume that $U=V \times \Delta$, where $V$ and $\Delta$ are open neighbourhoods of 0 in $\mathbf{K}^{n-1}$ and $\mathbf{K}$ (respectively), that $x_{0}=0$, and that

$$
f(x) \sim \tilde{x}^{\xi} l_{1}(x)^{m_{1}} \cdots l_{r}(x)^{m_{r}} g(x),
$$

$x=\left(x_{1}, \ldots, x_{n}\right)$, where:

$$
\tilde{x}=\left(x_{1}, \ldots, x_{n-1}\right) \text { and } \xi=\left(\xi_{1}, \ldots, \xi_{n-1}\right) \in \mathbf{N}^{n-1} .
$$

(3.6.2) $l_{i}(x)=x_{n}+a_{i}(\tilde{x}), i=1, \ldots, r$, where the $a_{i} \in \mathscr{O}(V)$ are distinct and $a_{i}(0)=0, i=1, \ldots, r$.

(3.6.3) $g(x)=x_{n}^{d}+\sum_{j=1}^{d} c_{j}(\tilde{x}) x_{n}^{d-j}$, where $d=d(0)$ and, for each $j=$ $1, \ldots, d, c_{j} \in \mathscr{O}(V)$ and $\mu_{0}\left(c_{j}\right) \geq j$.

(3.6.4) The $l_{i}, i=1, \ldots, r$, and the $x_{k}$ where $\xi_{k}>0, k=1, \ldots, n-1$, are precisely the irreducible factors of $D$. Moreover, none of these factors divides $g$ (in germs at 0 ).

We can, of course, find a local representation of $f$ as above, with $\xi=0$, but it is convenient to allow an exceptional factor $\tilde{x}^{\xi}$ in order to formulate an appropriate induction. The integer $r=r(0)$ in the representation of $f$ above depends on the coordinate system $\left(x_{1}, \ldots, x_{n}\right)$. However, if we consider the analogous representation of $f$ at points $x$ near 0 , in the coordinates obtained by translating the original coordinates to $x$, then the associated $r(x)$ is Zariski semicontinuous.

We will argue by induction on the triple $(n, d, r)$. (Triples $(n, d, r)$ are considered to be ordered lexicographically.)

If $n=1$, then $\mathcal{J}$ already has only normal crossings throughout $M$. If $d=0$, then $f(x)$ already has only normal crossings in a neighbourhood of 0 . Suppose that $d \geq 1$. Then, after a coordinate transformation

$$
\begin{aligned}
& x_{k}^{\prime}=x_{k}, \quad k=1, \ldots, n-1, \\
& x_{n}^{\prime}=x_{n}+\frac{1}{d} c_{1}(\tilde{x}),
\end{aligned}
$$


we can further assume that $c_{1}=0$; i.e.,

$$
g(x)=x_{n}^{d}+\sum_{j=2}^{d} c_{j}(\tilde{x}) x_{n}^{d-j} .
$$

The significance of this representation is that $\mu_{x}(g)=d, x=\left(x_{1}, \ldots, x_{n}\right)$, if and only if $x_{n}=0$ and $\mu_{\tilde{x}}\left(c_{j}\right) \geq j, j=2, \ldots, d$.

Now, if $r=0$ and $c_{j}=0, j=2, \ldots, d$, then $g(x)=x_{n}^{d}$ and $f(x)$ already has only normal crossings in a neighbourhood of 0 . Otherwise, at least one of the $a_{i}, i=1, \ldots, r$ or $c_{j}, j=2, \ldots, d$, is not identically zero. Let us assume that this is the case. We will show that, with $U$ small enough, there is a covering of $U$ by finitely many finite sequences of local blowingsup with smooth centres which are $\mu$-admissible with respect to the successive transforms of our data $\left(\left(f_{p}\right) ; f ; D\right)$, such that, for each sequence, the pairs $\left(d^{\prime}\left(x^{\prime}\right), r^{\prime}\left(x^{\prime}\right)\right)$ analogous to $(d, r)$ for the final transform $\left(\left(f_{p}^{\prime}\right) ; f^{\prime} ; D^{\prime}\right)$ in suitable local coordinates at each point $x^{\prime}$ where $\mu_{x^{\prime}}\left(f_{p}^{\prime}\right) \geq \mu, p=1, \ldots, s$, will be strictly less than $(d, r)$ (in the lexicographic ordering of such pairs). This will complete the proof.

Since $f_{1}, \ldots, f_{s}$ each divides $f$, the representation of $f$ above implies that, for each $p=1, \ldots, s$,

$$
f_{p}(x) \sim \tilde{x}^{\eta_{p}} \prod_{i=1}^{r} l_{i}(x)^{m_{p i}} \cdot g_{p}(x),
$$

where:

(3.8.1) $\eta_{p}=\left(\eta_{p 1}, \ldots, \eta_{p, n-1}\right) \in \mathbf{N}^{n-1}$ and $\eta_{p} \leq \xi$. (This means $\eta_{p k} \leq \xi_{k}$, $k=1, \ldots, n-1$.

$$
m_{p i} \leq m_{i}, i=1, \ldots, r \text {. }
$$

(3.8.3) $g_{p}$ divides $g$.

Let $d_{p}=\mu_{0}\left(g_{p}\right), p=1, \ldots, s$.

Remarks 3.9. Effect of blowing up. To elucidate the argument following, it is helpful to first compute the effect on our data of blowing up $U$ with centre satisfying certain conditions. Let $I \subset\{1, \ldots, n-1\}$. Let $\pi: U^{\prime} \rightarrow U$ denote the blowing-up with centre

$$
Z_{I}=\left\{x \in U: x_{n}=0 \text { and } x_{k}=0, k \in I\right\} .
$$

Then $U^{\prime}$ is covered by coordinate charts $U_{k}^{\prime}, k \in I \cup\{n\}$, where $\pi_{k}=\pi \mid U_{k}^{\prime}$ is given in coordinates $y=\left(y_{1}, \ldots, y_{n}\right)$ of $U_{k}^{\prime}$ by

$$
\begin{aligned}
x_{l} & =y_{l}, \quad l \notin I \cup\{n\}, \\
x_{k} & =y_{k}, \\
x_{l} & =y_{k} y_{l}, \quad l \in(I \cup\{n\})-\{k\} .
\end{aligned}
$$

If $y=\left(y_{1}, \ldots, y_{n}\right) \in U_{k}^{\prime}$, we write $\tilde{\pi}_{k}(\tilde{y})=\pi_{k}(y)^{\sim}$. 
Suppose that the centre $Z_{I}$ lies in the "equimultiple locus" of $l_{1}^{m_{1}} \ldots l_{r}^{m_{r}} g$; i.e., in the locus of points where this function has its maximum multiplicity $\sum_{i=1}^{r} m_{i}+d$. This equimultiple locus is given by the conditions

$$
\begin{aligned}
& x_{n}=0, \\
& \mu_{\tilde{x}}\left(a_{i}\right) \geq 1, \quad i=1, \ldots, r, \\
& \mu_{\tilde{x}}\left(c_{j}\right) \geq j, \quad j=2, \ldots, d .
\end{aligned}
$$

Then the composites $l_{i} \circ \pi$ and $g \circ \pi$ can be factored as analytic functions in the various coordinate charts of $U^{\prime}$, as follows. In $U_{n}^{\prime}$,

$$
\begin{aligned}
& \left(l_{i} \circ \pi_{n}\right)(y)=y_{n}\left(1+\frac{a_{i}\left(\tilde{\pi}_{n}(\tilde{y})\right)}{y_{n}}\right), \quad i=1, \ldots, r, \\
& \left(g \circ \pi_{n}\right)(y)=y_{n}^{d}\left(1+\sum_{j=2}^{d} \frac{c_{j}\left(\tilde{\pi}_{n}(\tilde{y})\right)}{y_{n}^{j}}\right) .
\end{aligned}
$$

In $U_{k}^{\prime}$, for fixed $k \in I$,

$$
\begin{aligned}
& \left(l_{i} \circ \pi_{k}\right)(y)=y_{k}\left(y_{n}+a_{i}^{\prime}(\tilde{y})\right), \quad i=1, \ldots, r, \\
& \left(g \circ \pi_{k}\right)(y)=y_{k}^{d}\left(y_{n}^{d}+\sum_{j=2}^{d} c_{j}^{\prime}(\tilde{y}) y_{n}^{d-j}\right),
\end{aligned}
$$

where

$$
\begin{array}{ll}
a_{i}^{\prime}(\tilde{y})=\frac{a_{i}\left(\tilde{\pi}_{k}(\tilde{y})\right)}{y_{k}}, & i=1, \ldots, r, \\
c_{j}^{\prime}(\tilde{y})=\frac{c_{j}\left(\tilde{\pi}_{k}(\tilde{y})\right)}{y_{k}^{j}}, & j=2, \ldots, d .
\end{array}
$$

(3.9.2) The formulas (3.9.1) imply that $f \circ \pi_{n}$ already has only normal crossings at every point of $U_{n}^{\prime}-\bigcup_{k \in I} U_{k}^{\prime}$. In particular, suppose there is a locally finite covering of $\bigcup_{k \in I} U_{k}^{\prime}$ satisfying the conclusion of Theorem 3.2. Then there is a locally finite covering of $U$ with the same property.

Now suppose that, in addition to the assumptions on $Z_{I}$ above,

$$
Z_{I} \subset\left\{x \in U: \mu_{x}\left(f_{p}\right) \geq \mu, p=1, \ldots, s\right\} .
$$

Then $Z_{I}$ is $\mu$-admissible. Since $Z_{I}$ lies in the equimultiple locus of $g$, it lies in the equimultiple locus of each $g_{p}$, by (3.8.3). Fix $k \in I$. For $y \in U_{k}^{\prime}$, write

$$
\begin{aligned}
& l_{i}^{\prime}(y)=y_{n}+a_{i}^{\prime}(\tilde{y}), \quad i=1, \ldots, r, \\
& g^{\prime}(y)=y_{n}^{d}+\sum_{j=2}^{d} c_{j}^{\prime}(\tilde{y}) y_{n}^{d-j}, \\
& g_{p}^{\prime}(y)=\frac{1}{y_{k}^{d}} g_{p}\left(\pi_{k}(y)\right), \quad p=1, \ldots, s .
\end{aligned}
$$


In $U_{k}^{\prime}$, the transforms $\left(\left(f_{p}^{\prime}\right) ; f^{\prime} ; D^{\prime}\right)$ of our data $\left(\left(f_{p}\right) ; f ; D\right)$ are given by

$$
f^{\prime}(y)=\left(f \circ \pi_{k}\right)(y)=\tilde{y}^{\xi^{\prime}} \prod_{i=1}^{r} l_{i}^{\prime}(y)^{m_{i}} g^{\prime}(y) \text {, }
$$

where $\xi^{\prime}=\left(\xi_{1}^{\prime}, \ldots, \xi_{n-1}^{\prime}\right)$ and

$$
\begin{aligned}
& \xi_{l}^{\prime}=\xi_{l}, \quad l \neq k, \\
& \xi_{k}^{\prime}=\sum_{l \in I} \xi_{l}+\sum_{i=1}^{r} m_{i}+d ; \\
& D^{\prime}(y)=y_{k}^{\mu}\left(D \circ \pi_{k}\right)(y) ; \\
& f_{p}^{\prime}(y)=\tilde{y}^{\eta_{p}^{\prime}} \prod_{i=1}^{r} l_{i}^{\prime}(y)^{m_{p i}} g_{p}^{\prime}(y), \quad p=1, \ldots, s,
\end{aligned}
$$

where $\eta_{p}^{\prime}=\left(\eta_{p 1}^{\prime}, \ldots, \eta_{p, n-1}^{\prime}\right), p=1, \ldots, s$, and

$$
\begin{aligned}
\eta_{p l}^{\prime} & =\eta_{p l}, \quad l \neq k, \\
\eta_{p k}^{\prime} & =\sum_{l \in I} \eta_{p l}+\sum_{i=1}^{r} m_{p i}+d_{p}-\mu .
\end{aligned}
$$

We now complete the proof of Theorem 3.2. Let $e=\max (d, \mu)$. Let us consider the following data,

$$
\left(\left(h_{q}\right)_{q=1, \ldots, t} ; h ; E\right),
$$

in one fewer variable $\tilde{x}=\left(x_{1}, \ldots, x_{n-1}\right) \in V$ :

(3.10.1) The $h_{q}(\tilde{x})$ are all the nonzero functions on $V$ in the following list:

$$
\begin{gathered}
a_{i}(\tilde{x})^{e !}, \quad i=1, \ldots, r ; \\
c_{j}(\tilde{x})^{\ell ! / j}, \quad j=2, \ldots, d ; \\
\left(\tilde{x}^{\eta_{p}}\right)^{e ! /\left(\mu-\sum_{i=1}^{r} m_{p i}-d_{p}\right)},
\end{gathered}
$$

for all $p$ such that $\mu-\sum_{i=1}^{r} m_{p i}-d_{p}>0$.

(3.10.2) $h(\tilde{x})$ is the product of all the functions $h_{q}(\tilde{x})$ and all of their nonzero differences.

$$
\text { (3.10.3) } \quad E(\tilde{x})=\prod_{\xi_{k} \neq 0} x_{k} \text {. }
$$

By induction on $n$, there is a locally finite covering of $V$ by finite sequences of local blowings-up with smooth centres which are $e$ !-admissible with respect to the successive transforms of the data above, such that, for each sequence, if $\left(\left(h_{q}^{\prime}\right) ; h^{\prime} ; E^{\prime}\right)$ denotes the (final) transform of the data, then $h^{\prime}$ has only normal crossings at every point where the multiplicity of each $h_{q}^{\prime}$ is at least $e$ !.

We will take the transforms of our original data $\left(\left(f_{p}\right) ; f ; D\right)$ by a locally finite covering of $U$ induced by that of $V$ above: Consider one of the local 
blowings-up involved in the locally finite covering of $V$; for simplicity, say the first, $\tilde{\pi}: V^{\prime} \rightarrow V$ (which we can assume covers all of $V$ ). Let $\widetilde{C}$ denote the centre of $\tilde{\pi}$. Let $\pi: U^{\prime} \rightarrow U$ be the blowing-up of $U$ with the same centre, considered as a subspace $C$ of $U$.

Since $\widetilde{C}$ is $e$ !-admissible for the data $\left(\left(h_{q}\right) ; h ; E\right)$, it follows that $C$ is $\mu$ admissible for $\left(\left(f_{p}\right) ; f ; D\right)$ : First, $C$ and $D$ simultaneously have only normal crossings because (by $(3.10 .3)$ and the fact that $D$ divides $f$ ) each factor $x_{k}$ of $D, k=1, \ldots, n-1$, divides $E$, and (since the functions $h_{q}$ include all nonzero $a_{i}^{e !}$, according to (3.10.1)) $C$ lies in the zero set of each factor $l_{i}$ of $D$. Secondly, the inclusion of all nonzero $a_{i}^{e !}$ and $c_{j}^{e ! / j}$ among the $h_{q}$ guarantees that $C$ lies in the equimultiple locus of each $l_{i}$ and of $g$ (by the representation (3.7)), hence also of each $g_{p}$ (by (3.8.3)). Therefore, for each $p$ such that $\sum_{i=1}^{r} m_{p i}+d_{p} \geq \mu, C \subset\left\{x: \mu_{x}\left(f_{p}\right) \geq \mu\right\}$. On the other hand, for each $p$ such that $\mu>\sum_{i=1}^{r} m_{p i}+d_{p}, \widetilde{C} \subset\left\{\tilde{x}: \mu_{\tilde{x}}\left(\tilde{x}^{\eta_{p}}\right) \geq \mu-\sum_{i=1}^{r} m_{p i}-d_{p}\right\}$, by (3.10.1), so that again $C \subset\left\{x: \mu_{x}\left(f_{p}\right) \geq \mu\right\}$.

After a change of coordinates in $V$, we can assume that $C$ is of the form $Z_{I}$, as in Remarks 3.9. Then (with the notation of Remarks 3.9), for each $k \in I, \pi \mid U_{k}^{\prime}$ is given by

$$
\pi\left(\tilde{y}, y_{n}\right)=\left(\tilde{\pi}(\tilde{y}), y_{k} y_{n}\right)
$$

On the other hand, $f \circ \pi$ already has only normal crossings at every point of $U_{n}^{\prime}-\bigcup_{k \in I} U_{k}^{\prime}$, so that condition (2) of Theorem 3.2 already holds for the transforms of our data in a neighbourhood of $U^{\prime}-\bigcup_{k \in I} U_{k}^{\prime}$ (cf. (3.9.2)).

Let $k \in I$. For each $y_{0} \in U_{k}^{\prime}$, let $\left(d^{\prime}\left(y_{0}\right), r^{\prime}\left(y_{0}\right)\right)$ denote the analogue of the pair $(d, r)$ for the data $\left(\left(f_{p}^{\prime}\right) ; f^{\prime} ; D^{\prime}\right)$ in the coordinates $\left(y_{1}, \ldots, y_{n}\right)$ of $U_{k}^{\prime}$, translated to $y_{0}$. From the formulas of Remarks 3.9, $\left(d^{\prime}\left(y_{0}\right), r^{\prime}\left(y_{0}\right)\right) \leq(d, r)$ and $\left(d^{\prime}\left(y_{0}\right), r^{\prime}\left(y_{0}\right)\right)=(d, r)$ only if $y_{0 n}=0$, where $y_{0}=\left(y_{01}, \ldots, y_{0 n}\right)$. In some neighbourhood of such a point, we can repeat the argument above for the subsequent local blowings-up involved in the locally finite covering of $V$. If $\left(d^{\prime}\left(y_{0}\right), r^{\prime}\left(y_{0}\right)\right)<(d, r)$, then, by induction on $(d, r)$, we can assume there is a locally finite covering of some neighbourhood of $y_{0}$ with respect to which the conclusion of the theorem holds. Finally, then, as a result of our induction on $n$, we can assume (that the nonzero functions in the following list satisfy):

$$
\begin{gathered}
a_{i}(\tilde{x})^{e !} \sim \tilde{x}^{\alpha_{i}}, \quad i=1, \ldots, r, \\
c_{j}(\tilde{x})^{e ! / j} \sim \tilde{x}^{\beta_{j}}, \quad j=2, \ldots, d, \\
\left(\tilde{x}^{\eta_{p}}\right)^{e ! /\left(\mu-\sum_{i} m_{p i}-d_{p}\right)} \sim \tilde{x}^{\gamma_{p}},
\end{gathered}
$$

for all $p$ such that $\mu-\sum_{i=1}^{r} m_{p i}-d_{p}>0$, where each $\alpha_{i}=\left(\alpha_{i 1}, \ldots, \alpha_{i, n-1}\right)$, $\beta_{j}=\left(\beta_{j 1}, \ldots, \beta_{j, n-1}\right)$, and $\gamma_{p}=\left(\gamma_{p 1}, \ldots, \gamma_{p, n-1}\right)$ belongs to $\mathbf{N}^{n-1}$; and 
(because of (3.10.2) and the elementary Lemma 3.12 following):

(3.11.2) $\left\{\alpha_{i}, \beta_{j}, \gamma_{p}\right\}$ is totally ordered with respect to the induced partial ordering from $\mathbf{N}^{n-1}\left(\delta \leq \varepsilon\right.$ means $\delta_{k} \leq \varepsilon_{k}, k=1, \ldots, n-1$, where $\delta=$ $\left(\delta_{1}, \ldots, \delta_{n-1}\right)$ and $\left.\varepsilon=\left(\varepsilon_{1}, \ldots, \varepsilon_{n-1}\right)\right)$.

Lemma 3.12. Let $z=\left(z_{1}, \ldots, z_{l}\right)$. Let $\alpha, \beta, \gamma \in \mathbf{N}^{l}$ and let $a(z), b(z)$, $c(z)$ be invertible elements of $\mathbf{K}\{z\}$. If

$$
a(z) z^{\alpha}-b(z) z^{\beta}=c(z) z^{\gamma}
$$

then either $\alpha \leq \beta$ or $\beta \leq \alpha$.

Proof. Put $\delta_{k}=\min \left(\alpha_{k}, \beta_{k}\right), k=1, \ldots, l$, where $\alpha=\left(\alpha_{1}, \ldots, \alpha_{l}\right), \beta=$ $\left(\beta_{1}, \ldots, \beta_{l}\right)$. Let $\delta=\left(\delta_{1}, \ldots, \delta_{l}\right)$. If $\delta=\alpha$, then $\alpha \leq \beta$. Otherwise, choose $k$ such that $\delta_{k} \neq \alpha_{k}$. Then, on $\left\{z: z_{k}=0\right\}$, we have $z^{\alpha-\delta}=0$ and $0 \neq-b(z) z^{\beta-\delta}=c(z) z^{\gamma-\delta}$. Since $b$ and $c$ are invertible, it follows that $\beta=\gamma$. Then $a(z) z^{\alpha}=(b(z)+c(z)) z^{\beta}$, so that $\beta \leq \alpha$.

To finish the proof of Theorem 3.2, let $\sigma$ denote the smallest among all the exponents $\alpha_{i}, \beta_{j}$, and $\gamma_{p}$; say, $\sigma=\left(\sigma_{1}, \ldots, \sigma_{n-1}\right)$. Then $|\sigma|=\sum_{k=1}^{n-1} \sigma_{k} \geq$ $e$ !. Put

$Z=\left\{x \in U: l_{i}(x)=0, i=1, \ldots, r ; \mu_{x}(g)=d ; \mu_{x}\left(f_{p}\right) \geq \mu, p=1, \ldots, s\right\}$.

Clearly,

$$
Z=\left\{x \in U: x_{n}=0 \text { and } \sum_{k \in J(\tilde{x})} \sigma_{k} \geq e !\right\},
$$

where $J(\tilde{x})=\left\{k: x_{k}=0, k=1, \ldots, n-1\right\}$. let $S$ denote the collection of minimal subsets $I$ of $\{1, \ldots, n-1\}$ such that $\sum_{k \in I} \sigma_{k} \geq e$ !, i.e., the collection of subsets $I$ of $\{1, \ldots, n-1\}$ such that

$$
0 \leq \sum_{k \in I} \sigma_{k}-e !<\sigma_{l},
$$

for all $l \in I$. For each $I \in S$, put

$$
Z_{I}=\left\{x \in U: x_{n}=0 \text { and } x_{k}=0, k \in I\right\} .
$$

The $Z_{I}, I \in S$, are the irreducible components of $Z$.

Let $I \in S$. Let $\pi: U^{\prime} \rightarrow U$ be the blowing-up with centre $Z_{I}$. (We use the notation of Remarks 3.9.) By (3.9.2), it is enough to consider $f \circ \pi_{k}$, where $\pi_{k}$ denotes $\pi$ restricted to the chart $U_{k}^{\prime}$, for each $k \in I$.

Fix $k \in I$. Consider the transforms $\left(\left(f_{p}^{\prime}\right) ; f^{\prime} ; D^{\prime}\right)$ of our data in the chart $U_{k}^{\prime}$. For each of the symbols we have associated to $\left(\left(f_{p}\right) ; f ; D\right)$, we will use the same symbol primed to denote the analogous notion for the transformed data $\left(\left(f_{p}^{\prime}\right) ; f^{\prime} ; D^{\prime}\right)$ in the coordinates $\left(y_{1}, \ldots, y_{n}\right)$ of $U_{k}^{\prime}$.

If $\left(d^{\prime}(y), r^{\prime}(y)\right)<(d, r)$ for all $y \in U_{k}^{\prime}$, then in this chart we are done. Suppose that $\left(d^{\prime}\left(y_{0}\right), r^{\prime}\left(y_{0}\right)\right)=(d, r)$, for some $y_{0} \in U_{k}^{\prime}, y_{0}=\left(y_{01}, \ldots, y_{0 n}\right)$. 
Then $y_{0 n}=0$ and, since the $a_{i}^{\prime}$ and $c_{j}^{\prime}$ are monomials (times invertible factors) in the coordinates of $U_{k}^{\prime},\left(d^{\prime}(0), r^{\prime}(0)\right)$ (or $\left(d^{\prime}, r^{\prime}\right)$ ) equals $(d, r)$.

We have

$$
\begin{aligned}
\alpha_{i l}^{\prime} & =\alpha_{i l}, \quad l \neq k, \\
\alpha_{i k}^{\prime} & =\sum_{l \in I} \alpha_{i l}-e ! ; \\
\beta_{j l}^{\prime} & =\beta_{j l}, \quad l \neq k, \\
\beta_{j k}^{\prime} & =\sum_{l \in I} \beta_{j l}-e ! .
\end{aligned}
$$

Suppose that $\left(d^{\prime}, r^{\prime}\right)=(d, r)$. Then each $d_{p}^{\prime}=d_{p}$ and each $m_{p i}^{\prime}=m_{p i}$, so that, for every $p=1, \ldots, s$,

$$
\mu-\sum_{i=1}^{r} m_{p i}^{\prime}-d_{p}^{\prime}=\mu-\sum_{i=1}^{r} m_{p i}-d_{p} .
$$

Therefore, for each $p$ such that $\mu-\sum_{i=1}^{r} m_{p i}-d_{p}>0$,

$$
\gamma_{p}^{\prime}=\frac{e ! \eta_{p}^{\prime}}{\mu-\sum_{i=1}^{r} m_{p i}-d_{p}},
$$

where $\eta_{p}^{\prime}$ is given by (3.9.3), so that

$$
\begin{aligned}
& \gamma_{p l}^{\prime}=\gamma_{p l}, \quad l \neq k, \\
& \gamma_{p k}^{\prime}=\sum_{l \in I} \gamma_{p l}-e !
\end{aligned}
$$

Thus,

$$
\begin{aligned}
\sigma_{l}^{\prime} & =\sigma_{l}, \quad l \neq k, \\
\sigma_{k}^{\prime} & =\sum_{l \in I} \sigma_{l}-e ! .
\end{aligned}
$$

In particular, $\left|\sigma^{\prime}\right|<|\sigma|$ (while $\left|\sigma^{\prime}\right| \geq e$ !).

In other words, either $\left(d^{\prime}, r^{\prime}\right)<(d, r)$ or $\left(d^{\prime}, r^{\prime}\right)=(d, r)$ and $e ! \leq\left|\sigma^{\prime}\right|<$ $|\sigma|$. In the latter case, it follows that, after at most $|\sigma| / e !+1$ local blowings-up over successive coordinate charts, as above, if $(d, r)$ has not decreased, then some $\eta_{p}$ where $\mu-\sum_{i=1}^{r} m_{p i}-d_{p}>0$ decreases to 0 . But this means that the transform of $f_{p}$ has multiplicity $\sum_{i=1}^{r} m_{p i}-d_{p}<\mu$ at 0 and hence throughout the chart. This completes the proof of Theorem 3.2.

Now let $X$ be a closed analytic hypersurface in $M$. Let $x_{0} \in X$. It is easy to see that if $\mu\left(\mathcal{F}_{X, x}\right)$ is locally constant on $X$ near $x_{0}$, then either $X_{\text {red }}$ is smooth at $x_{0}$ or $|X|$ has codimension at least 2 at $x_{0}$. (The latter can occur only if $\mathbf{K}=\mathbf{R}$.) On the other hand, suppose that $\mu\left(\mathscr{I}_{X, x}\right)$ is not locally constant on $X$ near $x_{0}$. Then $x_{0} \in \operatorname{Sing} X$. Moreover, if $x_{0}^{\prime} \in\left\{x: \mu\left(\mathscr{J}_{X, x}\right)=\right.$ $\mu\left(\mathscr{I}_{X, x_{0}}\right\}$ is close enough to $x_{0}$ and $\mu\left(\mathscr{I}_{X, x}\right)$ is locally constant near $x_{0}^{\prime}$, then $|X|$ has codimension at least 2 at $x_{0}^{\prime}$ (in particular, $x_{0}^{\prime} \in \operatorname{Sing} X_{\text {red }}$ ). 
Suppose that $X$ is a closed analytic hypersurface in $M$ and that $\mathscr{H}$ is a principal divisor on $M$ having only normal crossings. Let $U$ be an open subspace of $M$, and let $C$ be a smooth closed subspace of $U$. Suppose that $C$ satisfies the following two conditions:

(3.13.1) $\quad C$ and $\mathscr{H}$ simultaneously have only normal crossings.

(3.13.2) $C \subset X$ and $\mu\left(\mathscr{F}_{X, x}\right)$ is constant on $C$.

Let $\pi: U^{\prime} \rightarrow M$ denote the local blowing-up of $M$ over $U$ with centre $C$. We define a transformation $\left(X^{\prime}, \mathscr{H}^{\prime}\right)$ of $(X, \mathscr{H})$ by $\pi$ as follows: Let $X^{\prime}$ denote the strict transform of $X$ by $\pi$; i.e., $\mathscr{I}_{X^{\prime}}=\pi^{-1}\left(\mathscr{I}_{X}\right) \cdot \mathscr{K}^{-\mu}$, where $\mathscr{K}$ denotes the ideal sheaf of $\pi^{-1}(C)$ in $\mathscr{O}_{U^{\prime}}$ and $\mu=\mu\left(\mathscr{J}_{X, x}\right), x \in C$. Put $\mathscr{H}^{\prime}=\pi^{-1}(\mathscr{H}) \cdot \mathscr{K}^{\mu}$. Clearly, $\mu\left(\mathscr{I}_{X^{\prime}, x^{\prime}}\right) \leq \mu$, for all $x \in \pi^{-1}(C)$.

By (3.13.1), $\mathscr{H}^{\prime}$ has only normal crossings. Therefore, we can likewise define a transformation of the given data $(X, \mathscr{H})$ by a sequence of local blowingsup whose centres satisfy conditions (3.13.1) and (3.13.2) with respect to the successive transforms of $(X, \mathscr{H})$.

Definition 3.14. Suppose that $X_{\text {red }}$ is smooth and $\mu\left(\mathscr{F}_{X, x}\right)$ is locally constant on $X$. We will say that $C$ is (a)-admissible (as a centre of local blowing up over $U$ ) if $C$ satisfies conditions (3.13.1), (3.13.2) above and the following additional condition:

(a) $C$ is nowhere dense in $X$.

Proposition 3.15. Let $X$ be a closed analytic hypersurface in $M$, and let $\mathscr{H}$ be a principal divisor on $M$ having only normal crossings. Suppose that $X_{\text {red }}$ is smooth and $\mu\left(\mathscr{I}_{X, x}\right)$ is locally constant on $X$. Then there is a locally finite covering $\left\{\pi_{j}: M_{j} \rightarrow M\right\}$ of $M$ such that:

(1) Each $\pi_{j}$ is a composite of finitely many local blowings-up with smooth centres which are (a)-admissible with respect to the successive transforms of $(X, \mathscr{H})$.

(2) For each $j$, let $\left(X_{j}, \mathscr{H}_{j}\right)$ denote the (final) transform of $(X, \mathscr{H})$ by $\pi_{j}$. Then $X_{j, \text { red }}$ is smooth, $\mu\left(\mathscr{I}_{X_{j}, x^{\prime}}\right)$ is locally constant on $X_{j}$, and $X_{j}$ and $\mathscr{H}_{j}$ simultaneously have only normal crossings.

Proof. If $U$ is an open subspace of $M$ and $C$ is a smooth closed subspace of $U$ satisfying (3.13.1) and (3.13.2), then (locally) $C$ is nowhere dense in $X$ unless $X$ and $\mathscr{H}$ already simultaneously have only normal crossings. Clearly then, the result follows from Theorem 3.2 applied to the data $\left(\mathscr{I}_{X} ; \mathscr{I}_{X} \cdot \mathscr{H} ; \mathscr{H}\right)$, with $\mu$ the (locally) constant value of $\mu\left(\mathscr{I}_{X, x}\right)$.

Definition 3.16. We will say that $C$ is (b)-admissible (as a centre of local blowing-up over $U$ ) if $C$ satisfies conditions (3.13.1), (3.13.2) above and the following additional condition:

(b) For all $x_{0} \in C$, either $|X|$ has codimension at least 2 at $x_{0}$ or $\mu\left(\mathscr{I}_{X, x}\right)$ is not locally constant on $X$ near $x_{0}$. 
Theorem 3.17. Let $X$ be a closed analytic hypersurface in $M$, and let $\mathscr{H}$ be a principal divisor on $M$ having only normal crossings. Then there is a locally finite covering $\left\{\pi_{j}: M_{j} \rightarrow M\right\}$ of $M$ such that:

(1) Each $\pi_{j}$ is a composite of finitely many local blowings-up with smooth centres which are (b)-admissible with respect to the successive transforms of $(X, \mathscr{H})$.

(2) For each $j$, let $\left(X_{j}, \mathscr{H}_{j}\right)$ denote the (final) transform of $(X, \mathscr{H})$ by $\pi_{j}$. Then $X_{j, \text { red }}$ is smooth and $\mu\left(\mathscr{I}_{X_{j}, x^{\prime}}\right)$ is locally constant on $X_{j}$.

Proof. The theorem follows easily from the following weaker claim: There is a locally finite covering $\left\{\pi_{j}: M_{j} \rightarrow M\right\}$ of $M$ satisfying (1) and the following condition:

$\left(2^{\prime}\right)$ For each $j$, let $\left(X_{j}, \mathscr{H}_{j}\right)$ denote the transform of $(X, \mathscr{H})$ by $\pi_{j}$. Then, for each $x_{0}^{\prime} \in X_{j}$, either $X_{j \text {, red }}$ is smooth at $x_{0}^{\prime}$ and $\mu\left(\mathscr{F}_{X_{j}, x^{\prime}}\right)$ is locally constant on $X_{j}$ near $x_{0}^{\prime}$, or $\mu\left(\mathscr{I}_{X_{j}, x^{\prime}}\right)$ is not locally constant on $X_{j}$ near $x_{0}^{\prime}$ but $X_{j}$ and $\mathscr{H}_{j}$ simultaneously have only normal crossings at $x_{0}^{\prime}$.

Let $K$ be a compact subset of $M$. It suffices to prove the claim with $M$ replaced by some open neighbourhood $W$ of $K$. Let $\mu=\max \left\{\mu\left(\mathscr{J}_{X, x}\right): x \in\right.$ $K\}$. Clearly, we can assume that $\mu>0$ and that $K$ is small enough that one of the following conditions holds:

(i) $\mu\left(\mathscr{I}_{X, x}\right)=\mu$ and $X_{\text {red }}$ is smooth at $x$, for all $x \in X \cap K$.

(ii) For all $x_{0} \in K$ such that $\mu\left(\mathscr{F}_{X, x_{0}}\right)=\mu$, either $\mu\left(\mathscr{F}_{X, x}\right)$ is not locally constant near $x_{0}$ or $|X|$ has codimension at least 2 near $x_{0}$.

In case (i), there is nothing to do. In case (ii), by Theorem 3.2 applied to the data $\left(\mathscr{I}_{X} ; \mathscr{I}_{X} \cdot \mathscr{H} ; \mathscr{H}\right)$ in a suitable neighbourhood $W$ of $K$, there is a locally finite covering $\left\{\pi_{j}: W_{j} \rightarrow W\right\}$ of $W$ satisfying (1) and the following condition:

$\left(2^{\prime \prime}\right)$ For each $j$, let $\left(X_{j}, \mathscr{H}_{j}\right)$ denote the transform of $(X, \mathscr{H})$ by $\pi_{j}$. Then $\mu\left(\mathscr{I}_{X_{j}, x^{\prime}}\right) \leq \mu$, for all $x \in X_{j}$, and $X_{j}$ and $\mathscr{H}_{j}$ simultaneously have only normal crossings at every point $x_{0}^{\prime}$ where $\mu\left(\mathscr{I}_{X_{j}, x_{0}^{\prime}}\right)=\mu$.

The claim above follows by induction on $\mu$.

Theorem 3.17 and Proposition 3.15 together give a complete embedded uniformization statement in the case of a hypersurface. We obtain Theorem 1.1 in the hypersurface case from Theorem 3.17 in the case that $X$ is reduced and $\mathscr{H}=\mathscr{O}_{M}$.

\section{THE DIAGRAM OF INITIAL EXPONENTS}

Let $K$ be a field. Let $K \llbracket x \rrbracket$ denote the ring of formal power series in $x=\left(x_{1}, \ldots, x_{n}\right)$ with coefficients in $K$.

If $\alpha=\left(\alpha_{1}, \ldots, \alpha_{n}\right) \in \mathbf{N}^{n}$, put $|\alpha|=\alpha_{1}+\cdots+\alpha_{n}$. The lexicographic ordering of $(n+1)$-tuples $\left(|\alpha|, \alpha_{1}, \ldots, \alpha_{n}\right)$ induces a total ordering of $\mathbf{N}^{n}$. Let $f \in K \llbracket x \rrbracket$. Write $f=\sum_{\alpha \in \mathbf{N}^{n}} a_{\alpha} x^{\alpha}$, where $x^{\alpha}$ denotes $x_{1}^{\alpha_{1}} \cdots x_{n}^{\alpha_{n}}$. Let 
$\operatorname{supp} f=\left\{\alpha \in \mathbf{N}^{n}: f_{\alpha} \neq 0\right\}$. The initial exponent $\exp f$ of $f$ is defined as the smallest element of $\operatorname{supp} f$. If $\alpha=\exp f$, then $a_{\alpha} x^{\alpha}$ is called the initial monomial mon $f$ of $f$.

4.1. Hironaka's division algorithm. Let $g^{1}, \ldots, g^{t} \in K \llbracket x \rrbracket$, and let $\alpha_{i}=$ $\exp g^{i}, i=1, \ldots, t$. We associate to $\alpha_{1}, \ldots, \alpha_{t}$ the following decomposition of $\mathbf{N}^{n}$ : Set $\Delta_{0}=\varnothing$. Define

$$
\Delta_{i}=\left(\alpha_{i}+\mathbf{N}^{n}\right)-\bigcup_{j=0}^{i-1} \Delta_{j}, \quad i=1, \ldots, t .
$$

Put $\Delta=\mathbf{N}^{n}-\bigcup_{i=1}^{t} \Delta_{i}$.

Theorem 4.1.1 [2, Chapter $1, \S 1 ; 5$, Theorem 6.2]. (1) For every $f \in K \llbracket x \rrbracket$, there exist unique $q_{i} \in K \llbracket x \rrbracket, i=1, \ldots, t$, and $r \in K \llbracket x \rrbracket$ such that $\alpha_{i}+\operatorname{supp} q_{i} \subset$ $\Delta_{i}, i=1, \ldots, t, \operatorname{supp} r \subset \Delta$, and

$$
f=\sum_{i=1}^{t} q_{i} g^{i}+r
$$

(2) Suppose that $K=\mathbf{K}$ (i.e., $\mathbf{R}$ or $\mathbf{C}$ ) and that $f$ and $g^{1}, \ldots, g^{t}$ all converge. Then the $q_{i}$ and $r$ all converge.

Remark 4.1.2 [5, Remark 6.6]. Let $\mathfrak{m}$ denote the maximal ideal of $K \llbracket x \rrbracket$. In Theorem 4.1.1, if $k \in \mathbf{N}$ and $f \in \mathfrak{m}^{k}$, then $r \in \mathfrak{m}^{k}$ and each $q_{i} \in \mathfrak{m}^{k-\left|\alpha_{i}\right|}$ (where $\mathfrak{m}^{l}=K \llbracket x \rrbracket$ if $l \leq 0$ ).

4.2. The diagram of initial exponents. Let $\mathbf{K}\{x\}$ denote the ring of convergent power series in $x=\left(x_{1}, \ldots, x_{n}\right)$. Let $I$ be an ideal in $\mathbf{K} \llbracket x \rrbracket$ or $\mathbf{K}\{x\}$. The diagram of initial exponents $\mathfrak{N}(I) \subset \mathbf{N}^{n}$ is defined as

$$
\mathfrak{N}(I)=\{\exp g: g \in I\} \text {. }
$$

Clearly $\mathfrak{N}(I)+\mathbf{N}^{n}=\mathfrak{N}(I)$. If $I \subset \mathbf{K}\{x\}$, then $\mathfrak{N}(I)=\mathfrak{N}(I \cdot \mathbf{K} \llbracket x \rrbracket)$.

Suppose that $\mathfrak{N} \subset \mathbf{N}^{n}$ and $\mathfrak{N}+\mathbf{N}^{n}=\mathfrak{N}$. Then there is a smallest finite subset $\mathfrak{V}$ of $\mathfrak{N}$ such that $\mathfrak{N}=\mathfrak{V}+\mathbf{N}^{n}$. We call $\mathfrak{V}$ the vertices of $\mathfrak{N}$.

The following is a simple consequence of Theorem 4.1.1.

Corollary 4.2.1 [5, Corollary 6.8]. Let I be an ideal in $\mathbf{K} \llbracket x \rrbracket$ or $\mathbf{K}\{x\}$. Let $\alpha_{i}$, $i=1, \ldots, t$, denote the vertices of $\mathfrak{N}(I)$. Choose $g^{i} \in I$ such that $\alpha_{i}=\exp g^{i}$, $i=1, \ldots, t$, and let $\left\{\Delta_{i}, \Delta\right\}$ denote the decomposition of $\mathbf{N}^{n}$ determined by the $\alpha_{i}$, as in $\S 4.1$ above. Then

(1) $\mathfrak{N}(I)=\bigcup_{i} \Delta_{i}$ and the $g^{i}$ generate $I$.

(2) There is a unique set of generators $f^{i}$ of $I, i=1, \ldots, t$, such that, for each $i, \operatorname{supp}\left(f^{i}-x^{\alpha_{i}}\right) \subset \Delta$; in particular, mon $f^{i}=x^{\alpha_{i}}$.

We call $f^{1}, \ldots, f^{t}$ the standard basis of $I$.

Let $\mathscr{D}(n)=\left\{\mathfrak{N} \subset \mathbf{N}^{n}: \mathfrak{N}+\mathbf{N}^{n}=\mathfrak{N}\right\}$. If $\mathfrak{N} \in \mathscr{D}(n)$, let

$$
\mathbf{K}\{x\}^{\mathfrak{N}}=\{f \in \mathbf{K}\{x\}: \operatorname{supp} f \cap \mathfrak{N}=\varnothing\} .
$$


Clearly, $\mathbf{K}\{x\}^{\mathfrak{N}}$ is stable with respect to differentiation.

We totally order $\mathscr{D}(n)$ as follows. To each $\mathfrak{N} \in \mathscr{D}(n)$, we associate the sequence $v(\mathfrak{N})$ obtained by listing the vertices of $\mathfrak{N}$ in ascending order and completing this list to an infinite sequence by using $\infty$ for all the remaining terms. If $\mathfrak{N}^{1}, \mathfrak{N}^{2} \in \mathscr{D}(n)$, we say that $\mathfrak{N}^{1}<\mathfrak{N}^{2}$ provided that $v\left(\mathfrak{N}^{1}\right)<$ $v\left(\mathfrak{N}^{2}\right)$ with respect to the lexicographic ordering on the set of all such sequences. Clearly, if $\mathfrak{N}^{1} \supset \mathfrak{N}^{2}$, then $\mathfrak{N}^{1} \leq \mathfrak{N}^{2}$.

4.3. Variation of the diagram in parametrized families. Let $U$ be an open subset of $\mathbf{K}^{m}$, and let $X$ be a closed analytic subset of $U$. Let $\mathscr{O}(X) \llbracket y \rrbracket$ denote the ring of formal power series in $y=\left(y_{1}, \ldots, y_{n}\right)$ with coefficients in the ring $\mathscr{O}(X)$ of analytic functions on $X$.

Let $x \in X$. There is an evaluation mapping $g \mapsto g(x)$ of $\mathscr{O}(X)$ onto $\mathbf{K}$. If $f=\sum_{\alpha \in \mathbf{N}^{n}} f_{\alpha} y^{\alpha} \in \mathscr{O}(X) \llbracket y \rrbracket$, we write $f(x ; y)=\sum f_{\alpha}(x) y^{\alpha}$ when the coefficients are evaluated at $X$.

Example 4.3.1. Let $V$ be an open subset of $\mathbf{K}^{n}$, and let $\mathscr{O}=\mathscr{O}_{V}$. Let $\mathscr{I}$ be a coherent sheaf of ideals in $\mathscr{O}$. Suppose that $f_{1}, \ldots, f_{q} \in \mathscr{O}(V)$ generate $\mathscr{I}_{x}$, for all $x \in V$. For each $i=1, \ldots, q, f_{i}(x+y)=\sum_{\alpha} D^{\alpha} f_{i}(x) y^{\alpha} / \alpha$ !, where $D^{\alpha}=\partial^{|\alpha|} / \partial y_{1}^{\alpha_{1}} \cdots \partial y_{n}^{\alpha_{n}}$ and $\alpha !=\alpha_{1} ! \cdots \alpha_{n} !$. Then $f^{i}=\sum D^{\alpha} f_{i} \cdot y^{\alpha} / \alpha ! \epsilon$ $\mathscr{O}(V) \llbracket y \rrbracket$ and the $f^{i}(x ; y)$ generate $\mathscr{I}_{x}$, for all $x \in V$.

Theorem 4.3.2 [5, $\S 7 ; 6$, Theorem 4.4]. Let $f^{1}, \ldots, f^{q} \in \mathscr{O}(X) \llbracket y \rrbracket . \quad$ For each $x \in X$, let $\mathscr{J}_{x}$ denote the ideal in $\mathbf{K} \llbracket y \rrbracket$ generated by the $f^{i}(x ; y)$, $i=1, \ldots, q$, and put $\mathfrak{N}_{x}=\mathfrak{N}\left(\mathscr{J}_{x}\right)$. Then $\mathfrak{N}_{x}$, as a function $X \rightarrow \mathscr{D}(n)$, is Zariski semicontinuous.

\section{The Hilbert-SAMuel function}

Let $A$ be a Noetherian local ring. The Hilbert-Samuel function $H: \mathbf{N} \rightarrow \mathbf{N}$ of $A$ is defined by

$$
H(k)=\operatorname{dim}_{\mathbf{K}} \frac{A}{\mathfrak{m}^{k+1}}, \quad k \in \mathbf{N},
$$

where $\mathfrak{m}$ denotes the maximal ideal of $A$ and $K$ is the field $A / \mathfrak{m}$. Let $\mathbf{N}^{\mathbf{N}}$ denote the set of functions from $\mathbf{N}$ to itself. $\mathbf{N}^{\mathbf{N}}$ is partially ordered as follows. If $H, H^{\prime} \in \mathbf{N}^{\mathbf{N}}$, then $H<H^{\prime}$ if $H(k) \leq H^{\prime}(k)$ for all $k$, and $H(k)<H^{\prime}(k)$ for some $k$.

In this section, we prove three important theorems concerning the HilbertSamuel function:

(1) The Hilbert-Samuel function of the formal local ring associated to each ideal in a family parametrized as in $\S 4.3$ is Zariski semicontinuous on the space of parameters.

(2) Any decreasing sequence of Hilbert-Samuel functions associated to formal (or analytic) local rings stabilizes.

(3) For each point of an analytic space, the Hilbert-Samuel stratum coincides (locally) with the equimultiple locus of the standard basis of the defining ideal (with respect to any local embedding in affine space). 
5.1. Semicontinuity of the Hilbert-Samuel function. Let $A=\mathbf{K}\{x\}$ or $\mathbf{K} \llbracket x \rrbracket$, where $x=\left(x_{1}, \ldots, x_{n}\right)$, and let $I$ be an ideal in $A$. Let $H_{I}$ denote the Hilbert-Samuel function of $A / I$; i.e.,

$$
H_{I}(k)=\operatorname{dim}_{\mathbf{K}} \frac{A}{I+\mathfrak{m}^{k+1}}, \quad k \in \mathbf{N},
$$

where $\mathfrak{m}$ denotes the maximal ideal of $A$.

Lemma 5.1.1. For every $k \in \mathbf{N}, H_{I}(k)$ is the number of elements $\alpha \in \mathbf{N}^{n}$ such that $\alpha \notin \mathfrak{N}(I)$ and $|\alpha| \leq k$.

Proof. By Remark 4.1.2.

It follows from Lemma 5.1.1 that $H_{I}(k)$ coincides with a polynomial in $k$, for $k$ sufficiently large (the "Hilbert-Samuel polynomial").

Theorem 5.1.2. With the hypotheses of Theorem 4.3.2, let $H_{x}$ denote the HilbertSamuel function of $\mathbf{K} \llbracket y \rrbracket / \mathscr{J}_{x}, x \in X$. Then the function $x \mapsto H_{x}$ from $X$ to $\mathbf{N}^{\mathbf{N}}$ is Zariski semicontinuous.

Proof. It is easy to see that $H_{x}(k)$ is topologically upper semicontinuous, for each fixed $k$ (cf. [13, II.5.3]). The result then follows from Theorem 4.3.2.

Corollary 5.1.3. Let $M$ be a smooth analytic space and let $X$ be a closed analytic subspace of $M$. For each $x \in X$, let $H_{X, x}$ denote the Hilbert-Samuel function of $\mathscr{O}_{X, x}$. Then $H_{X, x}$ is Zariski semicontinuous on $X$.

Proof. Immediate. (See Example 4.3.1.)

5.2. The stability theorem. Let \#E denote the number of elements in a finite set $E$. For any $\mathfrak{N} \in \mathscr{D}(n)$, put

$$
H_{\mathfrak{N}}(k)=\#\left\{\alpha \in \mathbf{N}^{n}-\mathfrak{N}:|\alpha| \leq k\right\} .
$$

Theorem 5.2.1. Let $\mathfrak{N}^{j} \in \mathscr{D}(n), j=1,2, \ldots$ For each $j$, let $H_{j}=H_{\mathfrak{N}^{j}}$. If $H_{1} \geq H_{2} \geq \ldots$, then there exists $j_{0}$ such that $H_{j}=H_{j_{0}}$ when $j \geq j_{0}$.

Because of Lemma 5.1.1, Theorem 5.2.1 implies the following result.

Corollary 5.2.2. Let $I^{j}, j=1,2, \ldots$, be any sequence of ideals in $K \llbracket x \rrbracket$ (or $\mathbf{K}\{x\}), x=\left(x_{1}, \ldots, x_{n}\right)$. For each $j$, let $H_{j}$ denote the Hilbert-Samuel function $H_{I^{j}}$. If $H_{1} \geq H_{2} \geq \cdots$, then there exists $j_{0}$ such that $H_{j}=H_{j_{0}}$ when $j \geq j_{0}$.

Proof of Theorem 5.2.1. Suppose the theorem is false. Then we can assume that $\mathfrak{N}^{1} \neq \varnothing$ and that $H_{1}>H_{2}>\cdots$ (by passing to a subsequence if necessary). For each $j$, let $\alpha_{i}^{j}, i=1,2, \ldots$, denote the sequence of vertices of $\mathfrak{N}^{j}$ listed in ascending order and completed to an infinite sequence by using $\infty$ for all the remaining terms. Thus each $\alpha_{i}^{j}=\min \left\{\alpha \in \mathfrak{N}^{j}-\bigcup_{k<i}\left(\alpha_{k}^{j}+\mathbf{N}^{n}\right)\right\}$. Let $N_{0}$ denote the positive integers. We claim there is an infinite subsequence $j(s)$, $s=1,2, \ldots$, of $N_{0}$ such that $\alpha_{i}^{j(s)}$ is independent of $s$, say $\alpha_{i}^{j(s)}=\alpha_{i}$, when $s \geq i, i=1,2, \ldots$. Then $\bigcup_{i=1}^{\infty}\left(\alpha_{i}+\mathbf{N}^{n}\right) \in \mathscr{D}(n)$ and each $\alpha_{i} \notin \bigcup_{k<i}\left(\alpha_{k}+\mathbf{N}^{n}\right)$. 
Therefore, there exists $s_{0}$ such that $\alpha_{i}=\infty$ if $i \geq s_{0}$. Hence $H_{j(s)}=H_{j\left(s_{0}\right)}$, $s \geq s_{0}$; a contradiction.

We will find infinite subsequences $N_{1} \supset N_{2} \supset \cdots$ of $N_{0}$ satisfying the following condition: For each $s=1,2, \ldots$, say $N_{s}=\{j(s, t): t=1,2, \ldots\}$. Then $\alpha_{i}^{j(s, t)}$ is independent of $s$ and $t$, say $\alpha_{i}^{j(s, t)}=\alpha_{i}$, when $t \geq i, i=$ $1, \ldots, s$. The claim above follows taking $j(s)=j(s, s), s=1,2, \ldots$.

First, let $l=\left|\alpha_{1}^{1}\right|$. Then, for all $j, H_{j}(l) \leq H_{1}(l)<\#\left\{\alpha \in \mathbf{N}^{n}:|\alpha| \leq l\right\}$. Therefore each $\left|\alpha_{1}^{j}\right| \leq l$. Hence there are only finitely many possible values of $\alpha_{1}^{j} \in \mathbf{N}^{n}$. It follows that there is a subsequence $N_{1}=\{j(1, t): t=1,2, \ldots\}$ of $N_{0}$ such that $\alpha_{1}^{j(1, t)}$ is independent of $t$, say $\alpha_{1}^{j(1, t)}=\alpha_{1}$, for all $t$. By induction, assume that we have found $N_{1} \supset \cdots \supset N_{s}$ as above, for some $s \geq 1$. Then, for all $t=s, s+1, \ldots, \mathfrak{N}^{j(s, t)} \supset \bigcup_{i=1}^{s}\left(\alpha_{i}+\mathbf{N}^{n}\right)$, where each $\alpha_{i}=\alpha_{i}^{j(s, t)}$, $t \geq i$. Since $\mathfrak{N}^{j(s, s)} \neq \mathfrak{N}^{j(s, t)}, t>s$, it follows that $\alpha_{s+1}^{j(s, t)}<\infty$ for all $t>s$. Let $l=\left|\alpha_{s+1}^{j(s, s+1)}\right|$. Then

$$
H_{j(s, s+1)}(l)<\#\left\{\alpha \in \mathbf{N}^{n}-\bigcup_{i=1}^{s}\left(\alpha_{i}+\mathbf{N}^{n}\right):|\alpha| \leq l\right\} .
$$

Since $H_{j}(l) \leq H_{j(s, s+1)}(l)$ when $j \geq j(s, s+1)$, it follows that $\left|\alpha_{s+1}^{j(s, t)}\right| \leq l$, $t=s+1, s+2, \ldots$. Thus there are only finitely many possible values of $\alpha_{s+1}^{j(s, t)}$, $t>s$. It follows that there is a subsequence $N_{s+1}$ of $N_{s}$ as required.

5.3. The Hilbert-Samuel stratum and the equimultiple locus. Let $U$ be an open subspace of $\mathbf{K}^{n}$, and let $\mathscr{I}$ be a coherent sheaf of ideals in $\mathscr{O}=\mathscr{O}_{U}$. Fix $x_{0} \in U$. Let $S$ denote the Hilbert-Samuel stratum of $\mathscr{O} / \mathscr{I}$ containing $x_{0}$ :

$$
S=\left\{x \in U: H_{x}=H_{x_{0}}\right\},
$$

where $H_{x}$ denotes the Hilbert-Samuel function of $\mathscr{O}_{x} / \mathscr{I}_{x}, x \in U$.

For any $x \in U$, we can identify $\mathscr{O}_{x}$ with $\mathbf{K}\{y\}=\mathbf{K}\left\{y_{1}, \ldots, y_{n}\right\}$ by translating the affine coordinates of $\mathbf{K}^{n}$ to $x$. Suppose that $f_{i} \in \mathscr{O}_{x_{0}}, i=1, \ldots, t$, generate $\mathscr{I}_{x_{0}}$. There is a neighbourhood $V$ of $x_{0}$ in $U$ in which the $f_{i}$ all converge. Let $S_{\left(f_{i}\right)}$ denote the equimultiple locus,

$$
S_{\left(f_{i}\right)}=\left\{x \in V: \mu_{x}\left(f_{i}\right)=\mu_{x_{0}}\left(f_{i}\right), i=1, \ldots, t\right\} .
$$

Our aim in this subsection is to prove

Theorem 5.3.1. Suppose that $f^{1}, \ldots, f^{t}$ is the standard basis of $\mathscr{I}_{x_{0}} \subset \mathbf{K}\{y\}$. Then there is an open neighbourhood $V$ of $x_{0}$ in $U$ in which the $f^{i}$ all converge, and

$$
S=S_{\left(f^{i}\right)}
$$

Lemma 5.3.2. Let $V$ be a relatively compact open neighbourhood of $x_{0}$ in $U$. Then there exists $k_{0} \in \mathbf{N}$ such that if $x_{1}, x_{2} \in V$ and $H_{x_{1}}(k)=H_{x_{2}}(k)$ for all $k \leq k_{0}$, then $H_{x_{1}}=H_{x_{2}}$. 
Proof. This follows immediately from Zariski semicontinuity of the HilbertSamuel function.

We can assume that $U$ is an open neighbourhood of the origin and that $x_{0}=0$. Let $\mathfrak{N}=\mathfrak{N}\left(\mathscr{J}_{0}\right)$ and let $\alpha_{i} \in \mathbf{N}^{n}, i=1, \ldots, t$, denote the vertices of $\mathfrak{N}$. Define $\Delta_{i}, i=1, \ldots, t$, as in $\S 4.1$. For each $i, \Delta_{i}$ has the form $\Delta_{i}=\alpha_{i}+\square_{i}$, where $\square_{i} \subset \mathbf{N}^{n}$. Suppose that $f^{i} \in \mathscr{O}_{0}, i=1, \ldots, t$, and that $\exp f^{i}=\alpha_{i}$, for each $i$.

Lemma 5.3.3. Let $k_{0} \in \mathbf{N}$. Then there is an open neighbourhood $V$ of 0 in $U$ such that:

(1) Each $f^{i} \in \mathscr{O}(V)$. For any $P_{i} \in \mathbf{K}[y]$ such that degree $P_{i} \leq k_{0}, \quad i=$ $1, \ldots, t$, there exist $Q_{i} \in \mathscr{O}_{0}, i=1, \ldots, t$, such that, for each $i, Q_{i}$ converges in $V$ and $\operatorname{supp} Q_{i} \subset \square_{i}$, and, for all $x \in V$,

$$
\sum_{i=1}^{t} P_{i}(x) f^{i}(x)=\sum_{i=1}^{t} Q_{i}(x) f^{i}(x) .
$$

(2) For each $\gamma \in \mathbf{N}^{n}$ and $i=1, \ldots, t$, let $v_{\gamma, i}=v_{\gamma, i}(x ; y)$ denote $y^{\gamma} f^{i}(x+y) \in \mathscr{I}_{x} \subset \mathbf{K}\{y\}$, where $x \in V$. Then, for each $x \in V$ and $k \leq k_{0}$, the $v_{\gamma, i}(x ; y)$, where $\gamma \in \square_{i},|\gamma| \leq k-\mu_{0}\left(f^{i}\right)$ and $i=1, \ldots, t$, induce linearly independent elements of $\mathbf{K}\{y\} /(y)^{k+1}$. (Here $(y)$ denotes the maximal ideal of $\mathbf{K}\{y\}$.)

Proof. By Theorem 4.1.1, for each $\gamma \in \mathbf{N}^{n}$ and $i=1, \ldots, t$, there exist $q_{j}^{\gamma, i} \in \mathbf{K}\{y\}, j=1, \ldots, t$, such that $\operatorname{supp} q_{j}^{\gamma, i} \subset \square_{j}$ and

$$
y^{\gamma} f^{i}(y)=\sum_{j=1}^{t} q_{j}^{\gamma, i}(y) f^{j}(y) .
$$

The $f^{i}$ and the $q_{j}^{\gamma, i}$ such that $|\gamma| \leq k_{0}$ converge in a common neighbourhood $V$ of 0 ; (1) follows.

For each $k \in \mathbf{N}$, the $v_{\gamma, i}(x ; y)$, where $\gamma \in \square_{i},|\gamma| \leq k-\mu_{0}\left(f^{i}\right)$ and $i=1, \ldots, t$, induce a basis of the vector subspace

$$
\frac{\mathscr{I}_{x}+(y)^{k+1}}{(y)^{k+1}}
$$

of $\mathbf{K}\{y\} /(y)^{k+1}$ at $x=0$, hence induce linearly independent elements at nearby points $x$. It follows that we can choose $V$ satisfying (2) as well.

Corollary 5.3.4. Let $k_{0} \in \mathbf{N}$. Choose an open neighbourhood $V$ of 0 such that Lemma 5.3.3(1) holds and the $f^{i}$ generate $\mathscr{I}_{x}$, for all $x \in V$. Then, for all $k \leq k_{0}$ and $x \in V$,

$$
\mathscr{W}_{k}(x)+(y)^{k+1} \cap \mathscr{I}_{x}=\mathscr{I}_{x},
$$

where $\mathscr{W}_{k}(x)$ denotes the linear span in $\mathscr{O}_{x}$ of

$$
\left\{v_{\gamma, i}(x ; y): \gamma \in \square_{i},|\gamma| \leq k-\mu_{x}\left(f^{i}\right), i=1, \ldots, t\right\} .
$$


Proof. Let $k \leq k_{0}$ and $x \in V$. Suppose that $f \in \mathscr{I}_{x} \subset \mathbf{K}\{y\}$. Then there are polynomials $P_{i}(y)$ such that degree $P_{i} \leq k-\mu_{x}\left(f^{i}\right)$, for each $i$, and

$$
f(y)-\sum_{i=1}^{t} P_{i}(y) f^{i}(x+y) \in(y)^{k+1} \cap \mathscr{I}_{x} .
$$

By Lemma 5.3.3(1), there exist $Q_{i} \in \mathscr{O}_{0}, i=1, \ldots, t$, such that, for each $i$, $Q_{i}$ converges in $V$ and $\operatorname{supp} Q_{i} \subset \square_{i}$, and

$$
\sum_{i=1}^{t} P_{i}(y-x) f^{i}(y)=\sum_{i=1}^{t} Q_{i}(y) f^{i}(y) .
$$

Therefore,

$$
f(y)-\sum_{i=1}^{t} Q_{i}(x+y) f^{i}(x+y) \in(y)^{k+1} \cap \mathscr{I}_{x} .
$$

Since $\operatorname{supp} Q_{i}(x+y) \subset \square_{i}, i=1, \ldots, t$, the result follows.

Proposition 5.3.5. Let $V$ be a relatively compact neighbourhood of 0 in $U$ such that Lemma 5.3.3(1) and (2) hold with $k_{0}$ given by Lemma 5.3.2, and the $f^{i}$ generate $\mathscr{I}_{x}$, for all $x \in V$. Then $S_{\left(f^{i}\right)} \subset S$.

Proof. Let $x \in V$. Suppose that $\mu_{x}\left(f^{i}\right)=\mu_{0}\left(f^{i}\right), i=1, \ldots, t$. Then, if $k \leq k_{0}$,

$$
\begin{aligned}
\#\left\{\gamma \in \mathbf{N}^{n}:|\gamma| \leq k\right\}-H_{x}(k) & =\operatorname{dim}_{\mathbf{K}} \frac{\mathcal{I}_{x}+\mathfrak{m}_{x}^{k+1}}{\mathfrak{m}_{x}^{k+1}} \\
& =\sum_{i=1}^{t} \#\left\{\gamma \in \square_{i}:|\gamma| \leq k-\mu_{0}\left(f^{i}\right)\right\} \\
& =\#\left\{\gamma \in \mathbf{N}^{n}:|\gamma| \leq k\right\}-H_{0}(k),
\end{aligned}
$$

where the second equality holds by Lemma 5.3.3(2) and Corollary 5.3.4. The result follows, by Lemma 5.3.2.

Proof of Theorem 5.3.1. By Proposition 5.3.5, we can choose $V$ in which the $f^{i}$ all converge and $S_{\left(f^{i}\right)} \subset S$. We will prove that, after shrinking $V$ if necessary, $S \subset S_{\left(f^{i}\right)}$.

Let $\alpha_{i}=\exp f^{i}, i=1, \ldots, t$; in particular, $\left|\alpha_{i}\right|=\mu_{0}\left(f^{i}\right)$. We can assume that $\left|\alpha_{1}\right| \leq\left|\alpha_{2}\right| \leq \cdots \leq\left|\alpha_{t}\right|$. Associate to each $k \in \mathbf{N}$ the index $i(k)$ such that $\left|\alpha_{i}\right| \leq k$ precisely when $i=1, \ldots, i(k)$. For each $k$, the elements

$$
y^{\gamma} f^{i}(x+y), \quad \gamma \in \square_{i}, \quad|\gamma| \leq k-\mu_{0}\left(f^{i}\right), \quad 1 \leq i \leq i(k),
$$

and

$$
y^{\gamma}, \quad \gamma \notin \mathfrak{N}=\mathfrak{N}\left(\mathscr{J}_{0}\right), \quad|\gamma| \leq k,
$$

of $\mathbf{K}\{y\}$ induce a basis of $\mathbf{K}\{y\} /(y)^{k+1}$ when $x=0$. Choose $V$ small enough that they remain a basis for all $x \in V$. We can also take $V$ small enough that $\mu_{x}\left(f^{i}\right) \leq \mu_{0}\left(f^{i}\right), i=1, \ldots, t, x \in V$. 
Suppose $x \in S \cap V$. It suffices to show that $\mu_{x}\left(f^{i}\right) \geq \mu_{0}\left(f^{i}\right), i=1, \ldots, t$. Since $H_{x}=H_{0}$, it follows that, for each $k$, the $y^{\gamma} f^{i}(x+y)$ induce a basis of $\left(\mathscr{I}_{x}+(y)^{k+1}\right) /(y)^{k+1}$. In particular, for each $j=1, \ldots, t$, and each $k$,

$$
f^{j}(x+y)-\sum_{1 \leq i \leq i(k)}\left(\sum_{\substack{\gamma \in \square_{i} \\|\gamma| \leq k-\left|\alpha_{i}\right|}} c_{\gamma, i}^{j, k} \cdot y^{\gamma}\right) f^{i}(x+y) \in(y)^{k+1},
$$

where the $c_{\gamma, i}^{j, k} \in \mathbf{K}$. Fix $j$ and take $k=\left|\alpha_{j}\right|-1$. Since $f^{j}(y)-y^{\alpha_{j}} \in \mathbf{K}\{y\}^{\mathfrak{N}}$, then $f^{j}(x+y)-y^{\alpha_{j}} \in \mathbf{K}\{y\}^{\mathfrak{N}}$ and $f^{j}(x+y) \in \mathbf{K}\{y\}^{\mathfrak{N}}+(y)^{k+1}$. Therefore,

$$
\sum_{1 \leq i \leq i(k)}\left(\sum_{\substack{\gamma \in \square_{i} \\|\gamma| \leq k-\left|\alpha_{i}\right|}} c_{\gamma, i}^{j, k} \cdot y^{\gamma}\right) f^{i}(x+y) \in \mathbf{K}\{y\}^{\mathfrak{N}}+(y)^{k+1},
$$

which implies that all $c_{\gamma, i}^{j, k}=0$. Thus, $\mu_{x}\left(f^{j}\right) \geq\left|\alpha_{j}\right|$.

\section{ESSENTIAL VARIABLES OF A HOMOGENEOUS IDEAL}

Consider the space $\mathbf{K}^{n}$ with affine coordinate ring $\mathbf{K}[y]=\mathbf{K}\left[y_{1}, \ldots, y_{n}\right]$. Let $\mathfrak{m}$ denote the ideal in $\mathbf{K}[y]$ of polynomials which vanish at the origin of $\mathbf{K}^{n}$. By an affine coordinate system for $\mathbf{K}^{n}$, we mean any coordinate system $x=\left(x_{1}, \ldots, x_{n}\right)$ which differs from $y=\left(y_{1}, \ldots, y_{n}\right)$ by an invertible linear transformation $y=y(x)$. If $f(y) \in \mathbf{K}[y]$, we usually write $f(x)$ for $f(y(x))$.

Definition 6.1. Let $P_{i}(y)$ be a homogeneous polynomial of degree $d_{i}, i=$ $1, \ldots, t$. Suppose that there is an affine coordinate system $x=(w, z)$, where $w=\left(w_{1}, \ldots, w_{n-r}\right)$ and $z=\left(z_{1}, \ldots, z_{r}\right)$, with respect to which

$$
P_{i}(w, z)=P_{i}(z), \quad i=1, \ldots, t,
$$

and $r$ is minimal for such representations. Then we say that $\left(z_{1}, \ldots, z_{r}\right)$ is a system of essential variables for the $P_{i}$. We also say that each $z_{j}$ is an essential variable, or that the $P_{i}$ depend essentially on each $z_{j}$.

It is clear that if $\left(z_{1}, \ldots, z_{r}\right)$ is a system of essential variables for the $P_{i}$ and $x$ is any affine coordinate system of the form $x=(w, z), z=\left(z_{1}, \ldots, z_{r}\right)$, then $P_{i}(w, z)=P_{i}(z), i=1, \ldots, t$.

Lemma 6.2. Let $P_{i}(y)$ be a homogeneous polynomial of degree $d_{i}, i=1, \ldots, t$. Let $E$ denote the vector subspace of $\mathrm{m} / \mathrm{m}^{2}$ spanned by the partial derivatives $D^{\beta} P_{i}=\partial^{|\beta|} P_{i} / \partial y^{\beta},|\beta|=d_{i}-1, i=1, \ldots, t$. Then

(1) Let $E^{\perp}$ denote the vector subspace of the dual $\left(\mathrm{m} / \mathrm{m}^{2}\right)^{*}$ which is orthogonal to $E$. Then

$$
E^{\perp}=\left\{\xi \in\left(\mathfrak{m} / \mathfrak{m}^{2}\right)^{*}: \xi \cdot P_{i}=0, i=1, \ldots, t\right\} .
$$

$\left(\xi \in\left(\mathfrak{m} / \mathfrak{m}^{2}\right)^{*}\right.$ identifies with a derivation of $\mathbf{K}[y]$. In coordinates, $\xi$ is a directional derivative $\xi=\sum_{j=1}^{n} \xi_{j} \partial / \partial y_{j} ;$ i.e., $\xi \cdot P=\sum \xi_{j} \partial P / \partial y_{j}, P \in \mathbf{K}[y]$. In particular, $\xi \cdot P$ is independent of the affine coordinate system.) 
(2) $z=\left(z_{1}, \ldots, z_{r}\right)$ is a system of essential variables for the $P_{i}$ if and only if $z_{1}, \ldots, z_{r}$ induce a basis of $E$.

Proof. Write

$$
P_{i}(y)=\sum_{|\alpha|=d_{i}} c_{i, \alpha} \frac{y^{\alpha}}{\alpha !}, \quad i=1, \ldots, t .
$$

Then, for each $i$,

$$
\begin{array}{cc}
D^{\beta} P_{i}(y)=\sum_{j=1}^{n} c_{i, \beta+(j)} y_{j}, & |\beta|=d_{i}-1, \\
\frac{\partial P_{i}}{\partial y_{j}}(y)=\sum_{|\beta|=d_{i}-1} c_{i, \beta+(j)} \frac{y^{\beta}}{\beta !}, & j=1, \ldots, n,
\end{array}
$$

where $(j)$ denotes the multi-index with 1 in the $j$ th place and zeros elsewhere. Consider the matrix $C=\left(c_{i, \beta+(j)}\right)$ with rows indexed by $(i, \beta),|\beta|=d_{i}-1$, $i=1, \ldots, t$, and columns indexed by $j=1, \ldots, n$. Then each row of $C$ represents a linear form $D^{\beta} P_{i},|\beta|=d_{i}-1$, and $\operatorname{Ker} C \subset\left(\mathfrak{m} / \mathfrak{m}^{2}\right)^{*}$ is the space of directional derivatives which annihilate $P_{1}, \ldots, P_{t}$. This proves (1).

By (1), if $z_{1}, \ldots, z_{r}$ are linear combinations of the variables $y_{1}, \ldots, y_{n}$ which induce a basis of $E$, and $x$ is an affine coordinate system of the form $x=(w, z), z=\left(z_{1}, \ldots, z_{r}\right)$, then $P_{i}(x)=P_{i}(z), i=1, \ldots, t$. On the other hand, it is clear that if $(w, z)$ is an affine coordinate system such that the $P_{i}(w, z)$ depend only on $z=\left(z_{1}, \ldots, z_{r}\right)$, then the $z_{j}$ span a vector subspace of $\mathfrak{m} / \mathfrak{m}^{2}$ containing $E ;(2)$ follows.

Remark 6.3. $E^{\perp}$ identifies with the vector subspace of $\mathbf{K}^{n}$ along which the $P_{i}$ are invariant by translation; i.e., $\xi \in E^{\perp}$ if and only if $P_{i}(y+\xi)=P_{i}(y)$, $i=1, \ldots, t$. Clearly, $E^{\perp}$ is the locus of points where each $P_{i}$ has multiplicity $d_{i}$.

Examples 6.4. (1) Let $P\left(y_{1}, y_{2}\right)=y_{1}^{2}+2 y_{1} y_{2}+y_{2}^{2}$. After a change of variables $w=y_{1}-y_{2}, z=y_{1}+y_{2}$, we have $P(w, z)=z^{2}$; i.e., $P$ depends essentially on $z$.

(2) Suppose that $f \in \mathbf{K}\{y\}$ is of the form

$$
f(y)=y_{n}^{d}+\sum_{i=1}^{d} c_{i}(\tilde{y}) y_{n}^{d-i},
$$

where $\tilde{y}=\left(y_{1}, \ldots, y_{n-1}\right)$ and $\mu_{0}\left(c_{i}\right) \geq i, i=1, \ldots, d$. Then the linear part of $y_{n}+c_{1}(\tilde{y}) / d$ is an essential variable for the lowest order homogeneous part of $f(y)$ (cf. (3.7)).

Definition 6.5. Let $I \subset \mathbf{K}\{y\}$ be a homogeneous ideal (i.e., $I$ can be generated by homogeneous polynomials in $\left.y=\left(y_{1}, \ldots, y_{n}\right)\right)$. Suppose that $x=(w, z)$, where $w=\left(w_{1}, \ldots, w_{n-r}\right)$ and $z=\left(z_{1}, \ldots, z_{r}\right)$, is an affine coordinate system with respect to which we can choose a system of generators of $I$ depending only on $z$, and that $r$ is minimal for all such choices. Then we say 
that $\left(z_{1}, \ldots, z_{r}\right)$ is a system of essential variables for $I$. We also say that each $z_{j}$ is an essential variable, or that $I$ depends essentially on each $z_{j}$.

Remark 6.6. Let $I \subset \mathbf{K}\{x\}, x=\left(x_{1}, \ldots, x_{n}\right)$, be a homogeneous ideal. Clearly, the standard basis of $I$ consists of homogeneous polynomials. Suppose that $x=(w, z)$ where $w=\left(w_{1}, \ldots, w_{n-r}\right), z=\left(z_{1}, \ldots, z_{r}\right)$, and $I$ is generated by homogeneous polynomials $g_{i}(z)$ in $z$ alone. Let $I^{*} \subset \mathbf{K}\{z\}$ denote the ideal generated by the $g_{i}(z)$. Clearly, then:

(1) $\mathfrak{N}(I)=\mathbf{N}^{n-r} \times \mathfrak{N}\left(I^{*}\right)$.

(2) The standard basis of $I$ is the same as that of $I^{*}$ (considered as elements of $I$ ).

It follows, in particular, that if $I$ can be generated by convergent power series involving $z$ alone, then the standard basis of $I$ consists of homogeneous polynomials in $z$ alone.

Lemma 6.7. Let $I \subset \mathbf{K}\{y\}$ be a homogeneous ideal. Let $f^{i}(y), i=1, \ldots, t$, denote the standard basis of $I$. Say $d_{i}=$ degree $f^{i}(y), i=1, \ldots, t$. Let $E$ denote the vector subspace of $\mathfrak{m} / \mathfrak{m}^{2}$ spanned by the linear forms $D^{\beta} f^{i},|\beta|=$ $d_{i}-1, i=1, \ldots, t$ (where $\mathfrak{m}$ is the maximal ideal of $\mathbf{K}\{y\}$ ). Then:

(1) $E^{\perp}=\left\{\xi \in\left(\mathfrak{m} / \mathfrak{m}^{2}\right)^{*}: \xi \cdot f \in I\right.$, for all $\left.f \in I\right\}$. In particular, $E$ is independent of the coordinate system $y=\left(y_{1}, \ldots, y_{n}\right)$.

(2) $z=\left(z_{1}, \ldots, z_{r}\right)$ is a system of essential variables for $I$ if and only if $z_{1}, \ldots, z_{r}$ induce a basis of $E$.

(3) (The germ at 0 of) $E^{\perp}$ identifies with the Hilbert-Samuel stratum $S$ of $\mathbf{K}\{y\} / I$ containing 0 .

Proof. By Lemma 6.2(1), in order to prove (1) it suffices to show that if $\xi=$ $\sum_{j=1}^{n} \xi_{j} \partial / \partial y_{j} \in\left(\mathfrak{m} / \mathfrak{m}^{2}\right)^{*}$, then

$$
\sum_{j=1}^{n} \xi_{j} \frac{\partial f^{i}}{\partial y_{j}}=0, \quad i=1, \ldots, t
$$

if and only if

$$
\sum_{j=1}^{n} \xi_{j} \frac{\partial f}{\partial y_{j}} \in I, \quad \text { for all } f \in I .
$$

Assume (6.7.1). Let $f \in I$; say $f=\sum a_{i} f^{i}$, where each $a_{i} \in \mathbf{K}\{y\}$. Then

$$
\sum \xi_{j} \partial f / \partial y_{j}=\sum_{i, j} \xi_{j}\left(\partial a_{i} / \partial y_{j}\right) f^{i} \in I \text {. }
$$

Conversely, (6.7.2) implies that $\sum_{j} \xi_{j} \partial f^{i} / \partial y_{j} \in I, i=1, \ldots, t$. But

$$
\sum_{j} \xi_{j} \partial f^{i} / \partial y_{j} \in \mathbf{K}\{y\}^{\mathfrak{N}(I)}, \quad i=1, \ldots, t .
$$

Therefore, $\sum_{j} \xi_{j} \partial f^{i} / \partial y_{j}=0, i=1, \ldots, t$. 
(2) follows from (1), Lemma 6.2 and Remark 6.6. (3) follows from Theorem 5.3.1 and Remark 6.3.

\section{THE EFFECT OF BLOWING-UP}

Let $M$ be a smooth analytic space, and let $X$ be a closed analytic subspace of $M$. Let $U$ be an open subspace of $M$, and let $C$ be a smooth closed subspace of $U$. Denote by $\pi: U^{\prime} \rightarrow M$ the local blowing-up of $M$ over $U$ with centre $C$. Let $X^{\prime}$ denote the strict transform of $X$ by $\pi$ (cf. $\S 2.5$ ). Suppose that $x_{0} \in X \cap C$ and that $x_{0}^{\prime} \in \pi^{-1}\left(x_{0}\right)$. Put $I=\mathscr{I}_{X, x_{0}}, I^{\prime}=\mathscr{I}_{X^{\prime}, x_{0}^{\prime}}$. The notation of this paragraph will be fixed throughout the section.

Lemma 7.1. Suppose that $(w, z), w=\left(w_{1}, \ldots, w_{n-r}\right), z=\left(z_{1}, \ldots, z_{r}\right)$, is a local coordinate system in a neighbourhood of $x_{0}=0$, in which $C$ is defined by $z=0$. Let $f^{i}, i=1, \ldots, t$, denote the standard basis of $I \subset \mathbf{K}\{w, z\}$. If $\mu_{x_{0}}\left(f^{i}\right)=\mu_{C, x_{0}}\left(f^{i}\right), i=1, \ldots, t$, then $I^{\prime}$ is generated by the strict transforms $f^{i \prime}$ of the $f^{i}$ at $x_{0}^{\prime}$.

Proof. Put $\mu_{i}=\mu_{C, x_{0}}\left(f^{i}\right), i=1, \ldots, t$. Let $f \in I$, and let $\mu=\mu_{C, x_{0}}(f)$. It suffices to show that $f$ can be written as $f=\sum_{i=1}^{t} a_{i} f^{i}$, where each $a_{i} \in$ $\mathbf{K}\{w, z\}$ and $\mu_{C, x_{0}}\left(a_{i}\right) \geq \mu-\mu_{i}$. Let $\left\{\Delta_{i}\right\}$ denote the decomposition of $\mathfrak{N}(I)$ determined by $\alpha_{i}=\exp f^{i}, i=1, \ldots, t$, as in $\S 4.1$. By the division theorem, $f=\sum_{i=1}^{t} \alpha_{i} f^{i}$, where $a_{i} \in \mathbf{K}\{w, z\}$ and $\alpha_{i}+\operatorname{supp} a_{i} \subset \Delta_{i}, i=1, \ldots, t$. Let $\nu_{i}=\mu_{C, x_{0}}\left(a_{i}\right), i=1, \ldots, t$. Put $\nu=\min _{i}\left(\nu_{i}+\mu_{i}\right)$. If $g \in \mathbf{K}\{w, z\}$, let in $_{C} g$ denote the lowest order homogeneous part of $g$ as a power series in $z$ with coefficients in $\mathbf{K}\{w\}$. Then

$$
\sum_{\left\{i: \nu_{i}+\mu_{i}=\nu\right\}}\left(\operatorname{in}_{C} a_{i}\right)\left(\operatorname{in}_{C} f^{i}\right) \neq 0 .
$$

(Otherwise, since $\mu_{C, x_{0}}\left(f^{i}\right)=\mu_{x_{0}}\left(f^{i}\right), i=1, \ldots, t$, implies that $\exp$ in $_{C} f^{i}=$ $\alpha_{i}$, for each $i$, it follows from the uniqueness of division that $\operatorname{in}_{C} a_{i}=0$ for all $i$ such that $\nu_{i}+\mu_{i}=\nu$; a contradiction.) Therefore $\nu=\mu$ and $\nu_{i} \geq \mu-\mu_{i}$, $i=1, \ldots, t$.

If $f \in \mathbf{K}\{x\}, x=\left(x_{1}, \ldots, x_{n}\right)$, then the initial form in $f$ of $f$ means the lowest order homogeneous part of $f$. If $J \subset \mathbf{K}\{x\}$ is an ideal, then in $J$ denotes the homogeneous ideal generated by $\{$ in $f: f \in J\}$. Clearly, $\mathfrak{N}(J)=\mathfrak{N}($ in $J)$.

Suppose that $x=(w, z)$ is a local coordinate system in a neighbourhood of $x_{0}=0$ such that $z=\left(z_{1}, \ldots, z_{r}\right)$ is a system of essential variables for in $I$. Clearly, $\mathfrak{N}(I)=\mathfrak{N}($ in $I)$ has the form $\mathbf{N}^{n-r} \times \mathfrak{N}^{*}$, where $\mathfrak{N}^{*} \in \mathscr{D}(r)$. Let $f^{i}$, $i=1, \ldots, t$, denote the standard basis of $I \subset \mathbf{K}\{w, z\}$. Put $d_{i}=\mu_{x_{0}}\left(f^{i}\right)$, $i=1, \ldots, t$. Then we can write

$$
f^{i}(w, z)=\sum_{|\alpha|=d_{i}} a_{\alpha}^{i}(w, z)+\sum_{|\alpha|<d_{i}} a_{\alpha}^{i}(w) z^{\alpha},
$$


where $\mu_{0}\left(a_{\alpha}^{i}\right)>d_{i}-|\alpha|$ if $|\alpha|<d_{i}$, and

$$
\left(\text { in } f^{i}\right)(w, z)=\sum_{|\alpha|=d_{i}} a_{\alpha}^{i}(0,0) z^{\alpha},
$$

$i=1, \ldots, t$. In particular, we have:

Lemma 7.2. There is a local coordinate system $(w, z), w=\left(w_{1}, \ldots, w_{n-r}\right)$, $z=\left(z_{1}, \ldots, z_{r}\right)$, in a neighbourhood of $x_{0}=0$ with the following property: Let $f^{i}(w, z), i=1, \ldots, t$, denote the standard basis of $I \subset \mathbf{K}\{w, z\}$, and let $I^{*} \subset \mathbf{K}\{w, z\}$ denote the ideal generated by the $\left(\right.$ in $\left.f^{i}\right)(0, z)$. Then

(1) $\left(z_{1}, \ldots, z_{r}\right)$ is a system of essential variables for $I^{*}$.

(2) $\left(\right.$ in $\left.f^{i}\right)(0, z), i=1, \ldots, t$, is the standard basis of $I^{*}$.

(3) $\mathfrak{N}\left(I^{*}\right)=\mathfrak{N}(I)$.

Of course, we could have formulated a stronger statement. But the point of Lemma 7.2 is that it isolates those properties of $I$ which persist after local blowing up when the Hilbert-Samuel function does not decrease. The following is the key theorem of this section.

Theorem 7.3. Suppose that $C \subset S$ (in a neighbourhood of $x_{0}$ ), where $S$ denotes the Hilbert-Samuel stratum of $X$ containing $x_{0}$. Then:

(1) $H_{X^{\prime}, x_{0}^{\prime}} \leq H_{X, x_{0}}$.

(2) Suppose that $H_{X^{\prime}, x_{0}^{\prime}}=H_{X, x_{0}}$. Let $x=(w, z)$ be a local coordinate system as in Lemma 7.2. Then there is a local coordinate system $\left(w^{\prime}, z^{\prime}\right)$, where $z^{\prime}=\left(z_{1}^{\prime}, \ldots, z_{r}^{\prime}\right)$, in a neighbourhood of $x_{0}^{\prime}=0$, such that:

(i) The standard basis of $I^{\prime} \subset \mathbf{K}\left\{w^{\prime}, z^{\prime}\right\}$ consists of the strict transforms $f^{i \prime}$ of the $f^{i}$.

(ii) $\left(\right.$ in $\left.f^{i \prime}\right)(0, \cdot)=\left(\right.$ in $\left.f^{i}\right)(0, \cdot), i=1, \ldots, t$.

Conditions (i) and (ii) imply that $\mu_{x_{0}^{\prime}}\left(f^{i \prime}\right)=\mu_{x_{0}}\left(f^{i}\right), i=1, \ldots, t$, and $\mathfrak{N}\left(I^{\prime}\right)=\mathfrak{N}\left(I^{*}\right)$ (with the same $\left.I^{*} !\right)$.

Lemma 7.4. Let $x=(w, z)$ be a local coordinate system as in Lemma 7.2, and let $S$ denote the Hilbert-Samuel stratum of $X$ containing $x_{0}$. Then, after a local coordinate change of the form

$$
w^{\prime}=w, \quad z^{\prime}=z-\lambda(w),
$$

where $\lambda$ is analytic and $\lambda(0)=0$, we can assume additionally in Lemma 7.2 that $S \subset\{z=0\}$.

Proof. Let $\mathfrak{m}$ denote the maximal ideal of $\mathbf{K}\{x\}$. Let $f^{i}(w, z), i=1, \ldots, t$, denote the standard basis of $I$, and let $d_{i}=\mu_{x_{0}}\left(f^{i}\right), i=1, \ldots, t$. If $\alpha \in \mathbf{N}^{r}$, we write $D_{z}^{\alpha}=\partial^{|\alpha|} / \partial z^{\alpha}$. By Lemma 6.7 , the vector subspace of $\mathfrak{m} / \mathfrak{m}^{2}$ of linear forms in $z$ is generated by $r$ linear forms,

$$
\sum_{i, j} c_{k j} D_{z}^{\alpha_{j i}}\left(\text { in } f^{i}\right)(0, z), \quad k=1, \ldots, r,
$$


where $c_{k j} \in \mathbf{K}, \alpha_{j i} \in \mathbf{N}^{r}$, and $\left|\alpha_{j i}\right|=d_{i}-1$. Put

$$
g_{k}(w, z)=\sum_{i, j} c_{k j} D_{z}^{\alpha_{j i}} f^{i}(w, z), \quad k=1, \ldots, r .
$$

Then the determinant of the Jacobian matrix $\partial\left(g_{1}, \ldots, g_{r}\right) / \partial\left(z_{1}, \ldots, z_{r}\right)$ does not vanish at $(w, z)=(0,0)$. By the implicit function theorem, the system of equations $g_{k}(w, z)=0, k=1, \ldots, r$, can be solved as $z=\lambda(w)$, where $\lambda$ is analytic and $\lambda(0)=0$. Put $w^{\prime}=w$ and $z^{\prime}=z-\lambda(w)$. By Theorem 5.3.1, $S \subset\left\{z^{\prime}=0\right\}$. It is easy to see that the conditions of Lemma 7.2 are satisfied in the new coordinates $\left(w^{\prime}, z^{\prime}\right.$ ). (The exponent of any monomial in $\left(w^{\prime}, z^{\prime}\right)$ involving $w^{\prime}$ is larger than that of any monomial of the same degree not involving $w^{\prime}$.)

Proof of Theorem 7.3. Let $(w, z)$ be a local coordinate system as in Lemma 7.2. By Lemma 7.4, we can assume that $S \subset\{z=0\}$. Since $C \subset S$, we can further assume that $w=(x, y)$, where $x=\left(x_{1}, \ldots, x_{n-q-r}\right), y=\left(y_{1}, \ldots, y_{q}\right)$, and $C=\{y=z=0\}$.

Then $U^{\prime}$ can be covered by $q+r$ coordinate charts $U_{y_{j}}^{\prime}, j=1, \ldots, q$, and $U_{z_{k}}^{\prime}, k=1, \ldots, r$, as follows: Each $U_{y_{j}}^{\prime}$ has a coordinate system $\left(x^{\prime}, y^{\prime}, z^{\prime}\right)$, where $x^{\prime}=\left(x_{1}^{\prime}, \ldots, x_{n-q-r}^{\prime}\right), y^{\prime}=\left(y_{1}^{\prime}, \ldots, y_{q}^{\prime}\right)$, and $z^{\prime}=\left(z_{1}^{\prime}, \ldots, z_{r}^{\prime}\right)$, in which $\pi \mid U_{y_{j}}^{\prime}$ is given by

$$
x=x^{\prime}, \quad y_{j}=y_{j}^{\prime}, \quad y_{l}=y_{j}^{\prime} y_{l}^{\prime} \quad \text { if } l \neq j, \quad z=y_{j}^{\prime} z^{\prime} .
$$

In particular, $\pi^{-1}(C) \cap U_{y_{j}}^{\prime}=\left\{y_{j}^{\prime}=0\right\}$. Each $U_{z_{k}}^{\prime}$ has a coordinate system $\left(x^{\prime}, y^{\prime}, z^{\prime}\right)$ in which $\pi \mid U_{z_{k}}^{\prime}$ is given by

$$
x=x^{\prime}, \quad y=z_{k}^{\prime} y^{\prime}, \quad z_{k}=z_{k}^{\prime}, \quad z_{l}=z_{k}^{\prime} z_{l}^{\prime} \quad \text { if } l \neq k .
$$

In particular, $\pi^{-1}(C) \cap U_{z_{k}}^{\prime}=\left\{z_{k}^{\prime}=0\right\}$.

Case A. $x_{0}^{\prime} \in U_{y_{j}}^{\prime}$ for some $j$; say $j=1$. In the coordinates $\left(x^{\prime}, y^{\prime}, z^{\prime}\right)$ of $U_{y_{1}}^{\prime}$ above, we can write $x_{0}^{\prime}=(0, b, c)$, where $b=\left(0, b_{2}, \ldots, b_{q}\right), c=$ $\left(c_{1}, \ldots, c_{r}\right)$. If $y=\left(y_{1}, \ldots, y_{q}\right)$, put $\tilde{y}=\left(y_{2}, \ldots, y_{q}\right)$. Then $\pi \mid U_{y_{1}}^{\prime}$ can be written in coordinates $\left(x^{\prime}, y^{\prime}, z^{\prime}\right)$ centred at $x_{0}^{\prime}$ as

$$
x=x^{\prime}, \quad y_{1}=y_{1}^{\prime}, \quad \tilde{y}=y_{1}^{\prime}\left(\tilde{b}+\tilde{y}^{\prime}\right), \quad z=y_{1}^{\prime}\left(c+z^{\prime}\right) .
$$

Case B. $x_{0}^{\prime} \in U_{z_{k}}^{\prime}-\bigcup_{j=1}^{q} U_{y_{j}}^{\prime}$ for some $k$; say $k=1$. In the coordinates $\left(x^{\prime}, y^{\prime}, z^{\prime}\right)$ of $U_{z_{1}}^{\prime}$ above, we can write $x_{0}^{\prime}=(0,0, c)$, where $c=\left(0, c_{2}\right.$, $\left.\ldots, c_{r}\right)$. If $z=\left(z_{1}, \ldots, z_{r}\right)$, put $\tilde{z}=\left(z_{2}, \ldots, z_{r}\right)$. Then $\pi \mid U_{z_{1}}^{\prime}$ can be written in coordinates $\left(x^{\prime}, y^{\prime}, z^{\prime}\right)$ centred at $x_{0}^{\prime}$ as

$$
x=x^{\prime}, \quad y=z_{1}^{\prime} y^{\prime}, \quad z_{1}=z_{1}^{\prime}, \quad \tilde{z}=z_{1}^{\prime}\left(\tilde{c}+\tilde{z}^{\prime}\right) .
$$

In this case we will prove that $H_{I^{\prime}}<H_{I}$. 
Lemma 7.5. Let $f_{i}(u, v) \in \mathbf{K} \llbracket u, v \rrbracket, i=1, \ldots, t$, where $u=\left(u_{1}, \ldots, u_{n-r}\right)$, $v=\left(v_{1}, \ldots, v_{r}\right)$. Let $J_{1} \subset \mathbf{K} \llbracket u, v \rrbracket$ denote the ideal generated by the $f_{i}(u, v)$, and $J_{0} \subset \mathbf{K} \llbracket u, v \rrbracket$ the ideal generated by the $f_{i}(0, v)$. Then $H_{J_{1}} \leq H_{J_{0}}$.

Proof. For each $\lambda \in \mathbf{K}$, let $J(\lambda) \subset \mathbf{K} \llbracket u, v \rrbracket$ denote the ideal generated by the $f_{i}(\lambda u, v)$. If $\lambda \neq 0$, then $(u, v) \mapsto(\lambda u, v)$ induces an automorphism of $\mathbf{K} \llbracket u, v \rrbracket$ taking $J(1)$ onto $J(\lambda)$; in particular, $H_{J(\lambda)}=H_{J(1)}$. Therefore, by Theorem 5.1.2, $H_{J(1)} \leq H_{J(0)}$. But $J(1)=J_{1}$ and $J(0)=J_{0}$.

Lemma 7.6. Let $J$ be a homogeneous ideal in $\mathbf{K}\{x, y, z\}, x=\left(x_{1}, \ldots\right.$, $\left.x_{n-q-r}\right), y=\left(y_{1}, \ldots, y_{q}\right), z=\left(z_{1}, \ldots, z_{r}\right)$. Suppose that

$$
C \subset S \subset\{z=0\} \text {, }
$$

where $C$ is defined by $y=0, z=0$, and $S$ denotes the Hilbert-Samuel stratum of $J$ containing $x_{0}=0$ (i.e., $S$ is the Hilbert-Samuel stratum containing $x_{0}$ of the analytic subspace defined locally by $J$ ). Let $J^{\prime}$ denote the strict transform of $J$ at $x_{0}^{\prime}$. Then

(1) $H_{J^{\prime}} \leq H_{J}$.

(2) If $x_{0}^{\prime} \in U_{z_{k}}^{\prime}-\bigcup_{j=1}^{q} U_{y_{j}}^{\prime}$, for some $k$, then $H_{J^{\prime}}<H_{J}$.

Proof. The Hilbert-Samuel stratum $S$ identifies with the linear subspace $E^{\perp}$ defined as in Lemma 6.7. Therefore, since $C \subset S$, the standard basis of $J$ depends only on $(y, z)$. Write $v=(y, z), v=\left(v_{1}, \ldots, v_{q+r}\right)$, so that the charts $U_{y_{j}}^{\prime}, U_{z_{k}}^{\prime}$ defined above can be relabelled as $U_{v_{l}}^{\prime}$, defined analogously. Suppose that $x_{0}^{\prime} \in U_{v_{l}}^{\prime}$ for some $l$; say $l=1$. In the coordinates $\left(x^{\prime}, v^{\prime}\right)$ of $U_{v_{1}}^{\prime}$, we can write $x_{0}^{\prime}=(0, a)$, where $a=\left(0, a_{2}, \ldots, a_{q+r}\right)$. If $v=\left(v_{1}, \ldots, v_{q+r}\right)$, put $\tilde{v}=\left(v_{2}, \ldots, v_{q+r}\right)$. Then $\pi \mid U_{v_{1}}^{\prime}$ can be expressed in coordinates $\left(x^{\prime}, v^{\prime}\right)$ centred at $x_{0}^{\prime}$ as

$$
x=x^{\prime}, \quad v_{1}=v_{1}^{\prime}, \quad \tilde{v}=v_{1}^{\prime}\left(\tilde{a}+\tilde{v}^{\prime}\right) .
$$

If $P \in J \cap \mathbf{K}[v]$ is homogeneous of degree $d$, put

$$
P^{\prime}\left(v^{\prime}\right)=\frac{1}{\left(v_{1}^{\prime}\right)^{d}} P\left(v_{1}^{\prime}, v_{1}^{\prime}\left(\tilde{a}+\tilde{v}^{\prime}\right)\right)=P\left(1, \tilde{a}+\tilde{v}^{\prime}\right) \text {. }
$$

Then $P^{\prime}$ is the strict transform of $P$. The ideal $J^{\prime}$ is generated by the $P^{\prime}$, for all homogeneous $P \in J \cap \mathbf{K}[v]$. For each $\lambda \in \mathbf{K}$, let $J(\lambda) \subset \mathbf{K}\{x, v\}$ denote the ideal generated by $P\left(\lambda+v_{1}, \lambda \tilde{a}+\tilde{v}\right)$, for all homogeneous $P \in J \cap \mathbf{K}[v]$. By homogeneity, if $\lambda \neq 0$, then $v \mapsto \lambda v$ induces an automorphism of $\mathbf{K}[v]$ taking $J(\lambda)$ onto $J(1)$; in particular, $H_{J(\lambda)}=H_{J(1)}$. Therefore, by Theorem 5.1.2, $H_{J(1)} \leq H_{J(0)}$. But $J(0)=J$. On the other hand, if $P \in J \cap \mathbf{K}[v]$ is homogeneous of degree $d$, then

$$
P\left(1+v_{1},\left(1+v_{1}\right)(\tilde{a}+\tilde{v})\right)=\left(1+v_{1}\right)^{d} P(1, \tilde{a}+\tilde{v}) ;
$$

this shows that the isomorphism $x=x^{\prime}, v_{1}=v_{1}^{\prime}, \tilde{v}=\left(1+v_{1}^{\prime}\right)\left(\tilde{a}+\tilde{v}^{\prime}\right)-\tilde{a}$ (of germs at 0 ) takes $J^{\prime}$ to $J(1)$. In particular, $H_{J^{\prime}}=H_{J(1)}$. Thus $H_{J^{\prime}} \leq H_{J}$. 
The ideal $J(1)$ is the ideal generated by all homogeneous polynomials $P \in$ $J \cap \mathbf{K}[v]$ at the point $y_{0}=(x, v)$, where $x=0, v=(1, \tilde{a})$. Suppose that $x_{0}^{\prime} \in U_{z_{k}}^{\prime}-\bigcup_{j=1}^{q} U_{y_{j}}^{\prime}$ for some $k$; say $k=1$, so we can assume $v_{1}=z_{1}$. Then $y_{0}=(x, y, z)$, where $x=0, y=0$, and $z=(1, \tilde{c}), \tilde{c}=\left(c_{2}, \ldots, c_{r}\right)$. In particular, $y_{0} \notin\{z=0\}$. By Lemma 6.7(3), since $E^{\perp}=S \subset\{z=0\}$, $H_{J^{\prime}}=H_{J(1)}<H_{J}$.

We can now complete the proof of Theorem 7.3. Put $d_{i}=\mu_{x_{0}}\left(f^{i}\right), i=$ $1, \ldots, t$. For each $i$, we can write

$$
f^{i}(x, y, z)=\sum_{|\alpha|=d_{i}} a_{\alpha}^{i}(x, y, z) z^{\alpha}+\sum_{|\alpha|<d_{i}} a_{\alpha}^{i}(x, y) z^{\alpha},
$$

where

$$
\left(\text { in } f^{i}\right)(0,0, z)=\sum_{|\alpha|=d_{i}} a_{\alpha}^{i}(0,0,0) z^{\alpha}
$$

and, for all $\alpha$ such that $|\alpha|<d_{i}, \mu_{C, 0}\left(a_{\alpha}^{i}\right) \geq d_{i}-|\alpha|$ (since $\mu_{C, x_{0}}\left(f^{i}\right)=$ $\mu_{x_{0}}\left(f^{i}\right), i=1, \ldots, t$, by Theorem 5.3.1).

Case A. For each $i=1, \ldots, t$, the strict transform $f^{i \prime}$ of $f^{i}$ is given by

$$
\begin{aligned}
f^{i \prime}\left(x^{\prime}, y^{\prime}, z^{\prime}\right)= & \left(y_{1}^{\prime}\right)^{-d_{i}} f^{i}\left(x^{\prime}, y_{1}^{\prime}, y_{1}^{\prime}\left(\tilde{b}+\tilde{y}^{\prime}\right), y_{1}^{\prime}\left(c+z^{\prime}\right)\right) \\
= & \sum_{|\alpha|=d_{i}} a_{\alpha}^{i}\left(x^{\prime}, y_{1}^{\prime}, y_{1}^{\prime}\left(\tilde{b}+\tilde{y}^{\prime}\right), y_{1}^{\prime}\left(c+z^{\prime}\right)\right)\left(c+z^{\prime}\right)^{\alpha} \\
& +\sum_{|\alpha|<d_{i}} \frac{a_{\alpha}^{i}\left(x^{\prime}, y_{1}^{\prime}, y_{1}^{\prime}\left(\tilde{b}+\tilde{y}^{\prime}\right)\right)}{\left(y_{1}^{\prime}\right)^{d_{i}-|\alpha|}}\left(c+z^{\prime}\right)^{\alpha} .
\end{aligned}
$$

To show that $H_{I^{\prime}} \leq H_{I}$ : For each $i=1, \ldots, t$,

$$
\left(\text { in } f^{i}\right)(x, y, z)=\sum_{|\alpha|=d_{i}} a_{\alpha}^{i}(0) z^{\alpha}+\sum_{|\alpha|<d_{i}} a_{\alpha, d_{i}-|\alpha|}^{i}(y) z^{\alpha},
$$

where $a_{\alpha, d_{i}-|\alpha|}^{i}$ denotes the homogeneous part of $a_{\alpha}^{i}$ of order $d_{i}-|\alpha|$ (which depends on $y$ alone because $f^{i}$ has constant order $d_{i}$ on a neighbourhood of $x_{0}$ in $C$ ). Let $J=$ in $I$. Let $J^{\prime} \subset \mathbf{K}\left\{x^{\prime}, y^{\prime}, z^{\prime}\right\}$ denote the ideal generated by

$$
f^{\prime \prime}\left(0,0, \tilde{y}^{\prime}, z^{\prime}\right)=\sum_{|\alpha|=d_{i}} a_{\alpha}^{i}(0)\left(c+z^{\prime}\right)^{\alpha}+\sum_{|\alpha|<d_{i}} a_{\alpha, d_{i}-|\alpha|}^{i}\left(1, \tilde{b}+\tilde{y}^{\prime}\right)\left(c+z^{\prime}\right)^{\alpha},
$$

$i=1, \ldots, t$. Then $J^{\prime}$ is the strict transform of $J$ at $x_{0}^{\prime}$, by Lemma 7.1. By Lemma 7.5, $H_{I^{\prime}} \leq H_{J^{\prime}}$, and by Lemma 7.6, $H_{J^{\prime}} \leq H_{J}=H_{I}$.

For each $i, \exp f^{i}=\left(0,0, \alpha_{i}\right)$, where $\alpha_{i} \in \mathbf{N}^{r}$ and $\left|\alpha_{i}\right|=d_{i}$. The diagram $\mathfrak{N}(I)$ is of the form $\mathbf{N}^{n-r} \times \mathfrak{N}^{*}$, where $\mathfrak{N}^{*} \in \mathscr{D}(r)$. Therefore, the exponent of a given monomial $x^{*} y^{*} z^{\alpha}$ lies outside $\mathfrak{N}(I)$ if and only if $\alpha$ lies outside $\mathfrak{N}^{*}$. It follows that all exponents in the second sum in the formula for $f^{i \prime}\left(x^{\prime}, y^{\prime}, z^{\prime}\right)$ 
above lie outside $\mathfrak{N}(I)$, and all exponents in the first sum lie outside $\mathfrak{N}(I)$ except for that of mon $f^{i}=a_{\alpha_{i}}^{i}(0) z^{\alpha_{i}}$.

Since $C \subset S=S_{\left(f^{i}\right)}, \mu_{x_{0}^{\prime}}\left(f^{i \prime}\right) \leq d_{i}, i=1, \ldots, t$. If $\mu_{x_{0}^{\prime}}\left(f^{i \prime}\right)=d_{i}$, then mon $f^{i \prime}=a_{\alpha_{i}}^{i}(0) z^{\prime \alpha_{i}}$ and (in $\left.f^{i \prime}\right)\left(0, z^{\prime}\right)=\left(\right.$ in $\left.f^{i}\right)\left(0, z^{\prime}\right)$ (because any monomial of degree $d_{i}$ in $f^{i \prime}$ other than $a_{\alpha_{i}}^{i}(0) z^{\prime \alpha_{i}}$ either is in in $f^{i}$ and therefore has higher (lexicographic) order, or has a factor involving $x=\left(x_{1}, \ldots, x_{n-q-r}\right)$ or $y=\left(y_{1}, \ldots, y_{q}\right)$ and therefore also has higher lexicographic order). In particular, if $\mu_{x_{0}^{\prime}}\left(f^{i \prime}\right)=d_{i}, i=1, \ldots, t$, then $\mathfrak{N}\left(I^{\prime}\right) \supset \mathfrak{N}(I)$.

Suppose that $H_{I^{\prime}}=H_{I}$. We will prove that $\mu_{x_{0}^{\prime}}\left(f^{i \prime}\right)=d_{i}$, for each $i$, so that $\mathfrak{N}\left(I^{\prime}\right) \supset \mathfrak{N}(I)$. Then (by Lemma 5.1.1) $\mathfrak{N}\left(I^{\prime}\right)=\mathfrak{N}(I)$. It follows that $f^{i \prime}$, $i=1, \ldots, t$, is the standard basis of $I^{\prime}$. Thus we obtain the assertion (2) of the theorem.

We can assume that $\alpha_{1}<\alpha_{2}<\cdots<\alpha_{t}$ (with respect to the total ordering of $\left.\mathbf{N}^{r}\right)$. Take $1=i_{1}<i_{2}<\cdots<i_{m+1}=t+1$ such that, for each $l=1, \ldots, m$, $d_{i}=d_{i_{l}}$ if $i_{l} \leq i<i_{l+1}$ and $d_{i_{l+1}}>d_{i_{l}}$.

First consider $1=i_{1} \leq i<i_{2}$ (i.e., $\left|\alpha_{i}\right|=d_{1}$ ). Then $\mu_{x_{0}^{\prime}}\left(f^{i \prime}\right)=d_{1}$. (Otherwise, $H_{I^{\prime}}<H_{I}$, by Lemma 5.1.1.) By induction, assume that $l>1$ and $\mu_{x_{0}^{\prime}}\left(f^{i \prime}\right)=d_{i}, i=1, \ldots, i_{l}-1$. Since $H_{I^{\prime}}=H_{I}$ and $\operatorname{mon} f^{i \prime}=\operatorname{mon} f^{i}$, $i=1, \ldots, i_{l}-1$, it follows that

$$
\mathfrak{N}\left(I^{\prime}\right) \cap\left\{\gamma \in \mathbf{N}^{n}:|\gamma|<d_{i_{l}}\right\}=\mathfrak{N}(I) \cap\left\{\gamma \in \mathbf{N}^{n}:|\gamma|<d_{i_{l}}\right\} .
$$

Consider $i_{l} \leq i<i_{l+1}$. If $\mu_{x_{0}^{\prime}}\left(f^{i \prime}\right)<d_{i}=d_{i_{l}}$, then $\exp f^{i \prime} \in \mathfrak{N}(I) \cap\{\gamma:|\gamma|<$ $d_{i_{l}}$ \}. (Otherwise, $H_{I^{\prime}}<H_{I}$.) But this is impossible, as we have seen that $\exp f^{i \prime} \notin \mathfrak{N}(I)$ unless mon $f^{i \prime}=\operatorname{mon} f^{i}$. Therefore, $\mu\left(f^{i \prime}\right)=d_{i}, i=1, \ldots, t$. This completes Case A of the theorem.

Case B. For each $i=1, \ldots, t$, the strict transform $f^{i \prime}$ of $f^{i}$ is given by

$$
\begin{aligned}
f^{\prime \prime}\left(x^{\prime}, y^{\prime}, z^{\prime}\right)= & \left(z_{1}^{\prime}\right)^{-d_{i}} f^{i}\left(x^{\prime}, z_{1}^{\prime} y^{\prime}, z_{1}^{\prime}, z_{1}^{\prime}\left(\tilde{c}+\tilde{z}^{\prime}\right)\right) \\
= & \sum_{|\alpha|=d_{i}} a_{\alpha}^{i}\left(x^{\prime}, z_{1}^{\prime} y^{\prime}, z_{1}^{\prime}, z_{1}^{\prime}\left(\tilde{c}+\tilde{z}^{\prime}\right)\right)\left(\tilde{c}+\tilde{z}^{\prime}\right)^{\tilde{\alpha}} \\
& +\sum_{|\alpha|<d_{i}} \frac{a_{\alpha}^{i}\left(x^{\prime}, z_{1}^{\prime} y\right)}{\left(z_{1}^{\prime}\right)^{d_{i}-|\alpha|}}\left(\tilde{c}+\tilde{z}^{\prime}\right)^{\tilde{\alpha}}
\end{aligned}
$$

(where $\tilde{\alpha}=\left(\alpha_{2}, \ldots, \alpha_{r}\right)$ when $\left.\alpha=\left(\alpha_{1}, \ldots, \alpha_{r}\right)\right)$. Let $J=I^{*}$, the homogeneous ideal in $\mathbf{K}\{x, y, z\}$ generated by the (in $\left.f^{i}\right)(0,0, z)=\sum_{|\alpha|=d_{i}} a_{\alpha}^{i}(0) z^{\alpha}$. Let $J^{\prime} \subset \mathbf{K}\left\{x^{\prime}, y^{\prime}, z^{\prime}\right\}$ denote the ideal generated by

$$
f^{i \prime}\left(0,0,0, \tilde{z}^{\prime}\right)=\sum_{|\alpha|=d_{i}} a_{\alpha}^{i}(0)\left(\tilde{c}+\tilde{z}^{\prime}\right)^{\tilde{\alpha}}, \quad i=1, \ldots, t .
$$


Then $J^{\prime}$ is the strict transform of $J$ by $\pi$, at $x_{0}^{\prime}$. By Lemma 7.5, $H_{I^{\prime}} \leq H_{J^{\prime}}$, and by Lemma 7.6, $H_{J^{\prime}}<H_{J}=H_{I}$. This completes the proof of Theorem 7.3.

\section{EMBEDDED UNIFORMIZATION THEOREMS}

We show here how the main results of the previous sections can be used to deduce a general embedded uniformization theorem. There are many ways of formulating other desingularization problems which can be treated in the same way. Let $M$ be a smooth analytic space.

Remarks 8.1. Suppose that $X$ is a closed analytic subspace of $M$. Let $x_{0} \in$ $X$, and let $S$ denote the Hilbert-Samuel stratum $\left\{x_{0} \in X: H_{X, x}=H_{X, x_{0}}\right\}$. Suppose $x_{0} \in \operatorname{Sing} X$. Then (in some neighbourhood of $x_{0}$ ) $S \subset \operatorname{Sing} X$. In particular, if the germs at $x_{0}$ of $|X|$ and $|\operatorname{Sing} X|$ do not coincide (e.g., if $\mathbf{K}=\mathbf{C}$ and $X$ is reduced), then $H_{X, x}$ is not locally constant near $x_{0}$. Conversely, suppose that $H_{X, x}$ is not locally constant near $x_{0}$. Then $x_{0} \in$ Sing $X$. Moreover, if $x_{0}^{\prime} \in S$ is close enough to $x_{0}$ and $H_{X, x}$ is locally constant near $x_{0}^{\prime}$, then $x_{0}^{\prime} \in \operatorname{Sing} X_{\text {red }}$. (If $|X|$ were irreducible at $x_{0}$, then the dimension of $X$ at $x_{0}^{\prime}$ would be less than that at $x_{0}$. The statement follows because $H_{X, x_{0}}$ is greater than the Hilbert-Samuel function of any proper subspace of $X$ at $x_{0}$.)

Suppose that $X$ is a closed analytic subspace of $M$ and that $\mathscr{H}$ is a principal divisor on $M$ having only normal crossings. Let $U$ be an open subspace of $M$, and let $C$ be a smooth closed subspace of $U$. Suppose that $C$ satisfies the following two conditions:

(8.2.1) $C$ and $\mathscr{H}$ simultaneously have only normal crossings.

(8.2.2) ( $C \subset X$ and) $H_{X, x}$ is constant on $C$.

Condition (8.2.2) implies that $\mu\left(\mathscr{I}_{X, X}\right)$ is constant on $C$. Let $\pi: U^{\prime} \rightarrow$ $M$ denote the local blowing up of $M$ over $U$ with centre $C$. We define a transformation $\left(X^{\prime}, \mathscr{H}^{\prime}\right)$ of $(X, \mathscr{H})$ by $\pi$ as follows. Let $X^{\prime}$ be the strict transform of $X$ by $\pi$. Let $\mathscr{K}$ denote the ideal sheaf of $\pi^{-1}(C)$ in $\mathscr{O}_{U^{\prime}}$, and put $\mathscr{H}^{\prime}=\pi^{-1}(\mathscr{H}) \cdot \mathscr{K}^{\mu}$, where $\mu=\mu\left(\mathcal{F}_{X, x}\right), x \in C$.

By (8.2.1), $\mathscr{H}^{\prime}$ has only normal crossings. Therefore, we can likewise define a transformation $\left(X^{\prime}, \mathscr{H}^{\prime}\right)$ of the given data $(X, \mathscr{H})$ by a sequence of local blowings-up whose centres satisfy conditions $(8.2 .1)$ and (8.2.2) with respect to the successive transforms of $(X, \mathscr{H})$.

Definition 8.3. Suppose that $X_{\text {red }}$ is smooth and that $H_{X, x}$ is locally constant on $X$. We will say that $C$ is (a)-admissible (as a centre of local blowing up over $U$ ) if $C$ satisfies conditions (8.2.1) and (8.2.2) and the following additional condition:

(a) $C$ is nowhere dense in $X$. 
Theorem 8.4. Let $X$ be a closed analytic subspace of $M$, and let $\mathscr{H}$ be a principal divisor on $M$ having only normal crossings. Suppose that $X_{\text {red }}$ is smooth and $H_{X, x}$ is locally constant on $X$. Then there is a locally finite covering $\left\{\pi_{j}: M_{j} \rightarrow M\right\}$ of $M$ such that:

(1) Each $\pi_{j}$ is a composite of finitely many local blowings-up with smooth centres which are (a)-admissible with respect to the successive transforms of $(X, \mathscr{H})$.

(2) For each $j$, let $\left(X_{j}, \mathscr{H}_{j}\right)$ denote the (final) transform of $(X, \mathscr{H})$ by $\pi_{j}$. Then $X_{j, \text { red }}$ is smooth, $H_{X_{j}, x^{\prime}}$ is locally constant on $X_{j}$, and $X_{j}$ and $\mathscr{H}_{j}$ simultaneously have only normal crossings.

Proof. Let $x_{0} \in X$. Let $W$ be a local coordinate neighbourhood of $x_{0}=0$, and let $f^{1}, \ldots, f^{t}$ denote the standard basis of $\mathscr{I}_{X, x_{0}}$ (with respect to the coordinates of $W)$. Let $d_{i}=\mu_{x_{0}}\left(f^{i}\right), i=1, \ldots, t$. We can assume that $f^{i} \in \mathscr{O}(W), i=1, \ldots, t$, and (by Theorem 5.3.1) that $X$ coincides in $W$ with the equimultiple locus $\left\{x: \mu_{x}\left(f^{i}\right)=d_{i}, i=1, \ldots, t\right\}$. Let $\mathscr{I} \subset \mathscr{O}_{W}$ denote the principal divisor generated by $f^{1} \cdots f^{t}$. We apply Theorem 3.2 to the data $(\mathscr{I} ; \mathscr{I} \cdot \mathscr{H} ; \mathscr{H})$ on $W$, with $\mu=d_{1}+\cdots+d_{t}$. Let $\pi_{j}: W_{j} \rightarrow W$ be one of the sequences of local blowings-up given by Theorem 3.2; say $\pi_{j}=$ $\pi_{j 1} \circ \pi_{j 2} \circ \cdots \circ \pi_{j, k(j)}$, where, for each $k=1, \ldots, k(j), \pi_{j k}: W_{j k} \rightarrow W_{j, k-1}$ is a local blowing-up of $W_{j, k-1}$ with smooth centre $C_{j k}$, and $W_{j 0}=W$, $W_{j, k(j)}=W_{j}$. Suppose that $1 \leq k<k(j)$ and that $C_{j 1}, \ldots, C_{j k}$ are all (a)admissible with respect to the successive transforms of $(X, \mathscr{H})$. Let $\left(X_{j k}, \mathscr{H}_{j k}\right)$ denote the transform of $(X, \mathscr{H})$ by $\pi_{j 1} \circ \cdots \circ \pi_{j k}$. It follows that $X_{j k}$, red is smooth and (by Corollary 5.1.3 and Theorem 7.3(1)) that $H_{X_{j k}, x^{\prime}}$ is locally constant on $X_{j k}$. Let $x_{0}^{\prime} \in X_{j k}$. If $C_{j, k+1}$ coincides with $X_{j k}$ near $x_{0}^{\prime}$, then $X_{j k}$ and $\mathscr{H}_{j k}$ already simultaneously have only normal crossings near $x_{0}^{\prime}$. Therefore, we can assume that $C_{j, k+1}$ is (a)-admissible. The result follows from Theorem 3.2.

Definition 8.5. We will say that $C$ is (c)-admissible (as a centre of local blowing up over $U$ ) if $C$ satisfies conditions (8.2.1) and (8.2.2) and the following additional condition:

(c) For all $x_{0} \in C$, either $x_{0} \in \operatorname{Sing} X_{\text {red }}$ or $H_{X, x}$ is not locally constant on $X$ near $x_{0}$.

Theorem 8.6. Let $X$ be a closed analytic subspace of $M$, and let $\mathscr{H}$ be a principal divisor on $M$ which has only normal crossings. Then there is a locally finite covering $\left\{\pi_{j}: M_{j} \rightarrow M\right\}$ of $M$ such that

(1) Each $\pi_{j}$ is a composite of finitely many local blowings-up with smooth centres which are (c)-admissible with respect to the successive transforms of $(X, \mathscr{H})$.

(2) For each $j$, let $\left(X_{j}, \mathscr{H}_{j}\right)$ denote the (final) transform of $(X, \mathscr{H})$ by $\pi_{j}$. Then $X_{j, \text { red }}$ is smooth and $H_{X_{j}, x^{\prime}}$ is locally constant on $X_{j}$. 
Proof. We first prove the following weaker claim: There is a locally finite covering $\left\{\pi_{j}: M_{j} \rightarrow M\right\}$ satisfying (1) and the following condition:

$\left(2^{\prime}\right)$ For each $j$, let $\left(X_{j}, \mathscr{H}_{j}\right)$ denote the transform of $(X, \mathscr{H})$ by $\pi_{j}$. Then $H_{X_{j}, x^{\prime}}$ is locally constant on $X_{j}$.

Let $x_{0} \in X$. Suppose that $H_{X, x}$ is not locally constant on $X$ in a neighbourhood of $x_{0}$. Let $W$ be a local coordinate neighbourhood of $x_{0}=0$, as in Lemma 7.2, and let $f^{1}, \ldots, f^{t}$ denote the standard basis of $\mathscr{I}_{X, x_{0}}$ (with respect to the coordinates of $W$ ). We can assume that each $f^{i} \in \mathscr{O}(W)$ and (by Theorem 5.3.1) that $\left\{x \in W: H_{X, x}=H_{X, x_{0}}\right\}=\left\{x \in W: \mu_{x}\left(f^{i}\right)=\mu_{x_{0}}\left(f^{i}\right)\right.$, $i=1, \ldots, t\}$. We apply Theorem 3.17 to $(Y, \mathscr{H})$ on $W$, where $\mathscr{I}_{Y}$ is the principal divisor generated by $f^{1} \cdots f^{t}$. Let $\pi_{j}^{\prime}: W_{j} \rightarrow W$ be one of the sequences of local blowings-up given by Theorem 3.17, say $\pi_{j}^{\prime}=\pi_{j 1} \circ \cdots \circ \pi_{j, k(j)}$, where, for all $k=1, \ldots, k(j), \pi_{j k}: W_{j k} \rightarrow W_{j, k-1}$ is a local blowing-up of $W_{j, k-1}$ with smooth centre $C_{j k}$, and $W_{j 0}=W, W_{j, k(j)}=W_{j}$.

Suppose that $1 \leq k<k(j)$ and that $C_{j 1}, \ldots, C_{j k}$ are all (c)-admissible with respect to the successive transforms of $(X, \mathscr{H})$. For each $l=1, \ldots, k$, let $\left(X_{j l}, \mathscr{H}_{j l}\right)$ denote the transform of $(X, \mathscr{H})$ by $\pi_{j}^{l}=\pi_{j 1} \circ \cdots \circ \pi_{j l}$, and let $Y_{j l}$ denote the strict transform of $Y$ by $\pi_{j}^{l}$. Suppose that $x_{0 l} \in X_{j l}, l=$ $1, \ldots, k$, and that $\pi_{j l}\left(x_{0 l}\right)=x_{0, l-1}, l=1, \ldots, k$, where $x_{00}=x_{0} \in C_{j 1}$. If $H_{X_{j l}, x_{0 l}}=H_{X, x_{0}}, l=1, \ldots, k$, then (by Theorem 7.3) $\mu\left(\mathscr{I}_{Y_{j l}, x_{0 l}}\right)=\mu\left(\mathscr{F}_{Y, x_{0}}\right)$, $l=1, \ldots, k$, and (using Theorem 5.3.1) the Hilbert-Samuel stratum of $X_{j k}$ containing $x_{0 k}$ coincides near $x_{0 k}$ with the equimultiple locus through $x_{0 k}$ of the strict transforms of the $f^{i}$. Suppose that $x_{0 k} \in C_{j, k+1}$. Unless $H_{X_{j k}, x^{\prime}}$ is already locally constant near $x_{0 k}$, it follows that $C_{j, k+1}$ is (c)-admissible near $x_{0 k}$.

It follows from Theorem 3.17 and Corollary 5.1.3 that there is a locally finite covering $\left\{\pi_{j}^{\prime \prime}: M_{j}^{\prime \prime} \rightarrow M\right\}$ satisfying (1) and the following condition:

$\left(2^{\prime \prime}\right)$ For all $j$, let $\left(X_{j}, \mathscr{H}_{j}\right)$ denote the transform of $(X, \mathscr{H})$ by $\pi_{j}^{\prime \prime}$. If $x_{0} \in X$ and $H_{X, x}$ is not locally constant near $x_{0}$, then $H_{X_{j}, x_{0}^{\prime}}<H_{X, x_{0}}$, for all $x_{0}^{\prime} \in\left(\pi_{j}^{\prime \prime}\right)^{-1}\left(x_{0}\right) \cap X_{j}$.

Our claim then follows by Corollary 5.2.2.

On the other hand, if $X$ is reduced, then the conclusion of the theorem follows from precisely the argument of the claim. (If $X$ is not smooth at $x_{0}$, then $C_{j, k+1}$ is (c)-admissible near $x_{0 k}$ unless $X_{j k}$ is already smooth at $x_{0 k}$. ) Therefore (because of Corollary 5.1.3 and Theorem 7.3(1)) we obtain the theorem by first applying the claim to $X$ and then the same argument to each $X_{j, \text { red }}$.

By applying Theorem 8.4 to each $\left(X_{j}, \mathscr{H}_{j}\right)$ in the conclusion of Theorem 8.6, we obtain a strong embedded uniformization theorem. Theorem 1.1 follows from Theorem 8.6 in the case that $X$ is reduced and $\mathscr{H}=\mathscr{O}_{M}$. 


\section{BIBLIOGRAPHY}

1. S. S. Abhyankar, Weighted expansions for canonical desingularization, Lecture Notes in Math., no. 910, Springer, Berlin, Heidelberg, and New York, 1982.

2. J. M. Aroca, H. Hironaka, and J. L. Vicente, The theory of the maximal contact, Mem. Mat. Inst. Jorge Juan, no. 29, Consejo Superior de Investigaciones Científicas, Madrid, 1975.

3. __ Desingularization theorems, Mem. Mat. Inst. Jorge Juan, no. 30, Consejo Superior de Investigaciones Científicas, Madrid, 1977.

4. B. M. Bennet, On the characteristic functions of a local ring, Ann. of Math. (2) 91 (1970), 25-87.

5. E. Bierstone and P. D. Milman, Relations among analytic functions I, Ann. Inst. Fourier (Grenoble) 37 (1) (1987), 187-239.

6. I Ideals of holomorphic functions with $\mathscr{C}^{\infty}$ boundary values on a pseudoconvex domain, Trans. Amer. Math. Soc. 304 (1987), 323-342.

7. __ Semianalytic and subanalytic sets, Inst. Hautes Études Sci. Publ. Math. 67 (1988), 5-42.

8. H. Hironaka, Resolution of singularities of an algebraic variety over a field of characteristic zero: I, II, Ann. of Math. (2) 79 (1964), 109-326.

9. __ Certain numerical characters of singularities, J. Math. Kyoto Univ. 10 (1970), 151-187.

10. __ Introduction to the theory of infinitely near singular points, Mem. Mat. Inst. Jorge Juan, no. 28, Consejo Superior de Investigaciones Científicas, Madrid, 1974.

11. __ Idealistic exponents of singularity, Algebraic Geometry (J. J. Sylvester Sympos., Johns Hopkins Univ., Baltimore, 1976), John Hopkins Univ. Press, Baltimore, 1977, pp. 52-125.

12. M. Spivakovsky, A solution to Hironaka's polyhedra game, Arithmetic and geometry, Vol. II, Progr. Math., no. 36, Birkhäuser, Boston, 1983, pp. 419-432.

13. J. C. Tougeron, Idéaux de fonctions différentiables, Springer, Berlin, Heidelberg, and New York, 1972.

14. B. Youssin, Newton polyhedra without coordinates, Mem. Amer. Math. Soc. (to appear).

15. _ Newton polyhedra of ideals, Mem. Amer. Math. Soc. (to appear).

16. O. Zariski, Local uniformization on algebraic varieties, Ann. of Math. (2) 41 (1940), 852-896.

Centre National de la Recherche Scientifique, Université de Paris Vi, 75005 Paris, FRANCE

Institut des Hautes Études Scientifiques, 91440 Bures-sur-Yvette, France

Department of Mathematics, University of Toronto, Toronto, Ontario, Canada M5S $1 \mathrm{~A} 1$

Current address of both authors: Department of Mathematics, University of Toronto, Toronto, Canada M5S 1A1 